\title{
Relações entre desigualdades de Poincaré ponderadas e existência de fórmulas de representação para campos vetoriais
}

\author{
Sílvia Lopes de Sena"
}

Orientador: Prof. Dr. Sérgio Luis Zani

Dissertação apresentada ao Instituto de Ciências Matemáticas e de Computação - ICMC-USP, como parte dos requisitos para obtenção do título de Mestre em Matemática.

USP - São Carlos

$\mathrm{Março/2001}$

"Este trabalho contou com apoio financeiro da CAPES 
A Comissão Julgadora:

Prof. Dr. Sérgio Luis Zani

Prof. Dr. Wagner Vieira Leite Nunes

Prof. Dr. Pedro Luiz Aparecido Malagutti

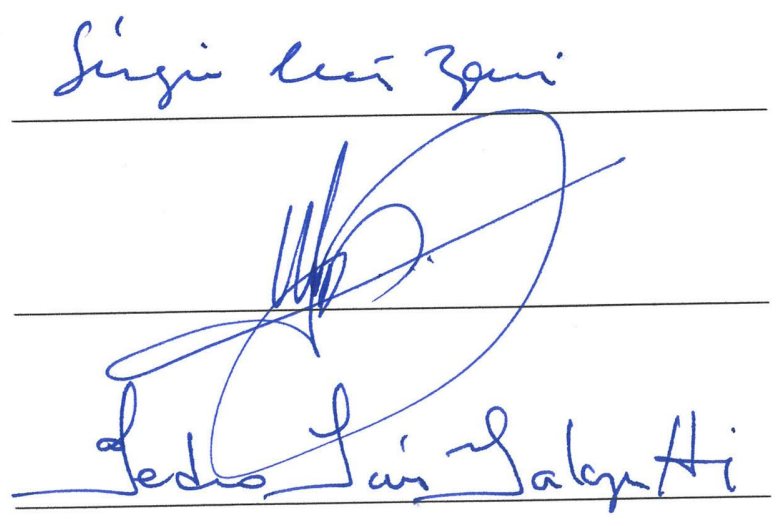


"À minha família e ao Júnior."

Muitos são os propósitos no coração do homem, mas é a vontade do Senhor que se realiza.

(Pv. 19:21) 


\section{Resumo}

Neste trabalho estudamos a relação entre as desigualdades ponderadas de Poincaré

em $L^{1}$ e a existência de fórmulas de representação envolvendo campos vetoriais de primeira ordem. 


\section{Agradecimentos}

A Deus por mais essa Graça em minha vida. O meu coração se alegra em Ti, ó Senhor meu.

Sou imensamente grata ao Prof. Dr. Sérgio Luís Zani, meu orientador, não só pela transmissão do conhecimento científico e por ser um excelente profissional, mas também pela dedicação e amizade. Este trabalho também é seu!!

Aos professores do ICMC, em especial ao Alexandre Nolasco de Carvalho por ter me encorajado e feito acreditar que nada é impossível quando existe um mínimo de esforço. À Maria do Carmo Carbinatto, orientadora de programa, e à Ires Dias a qual tenho um carinho muito grande.

Também não poderia deixar de agradecer os professores da UNESP de Presidente Prudente, especialmente ao Prof. Luiz F. Galante por ter me incentivado a inscrever-

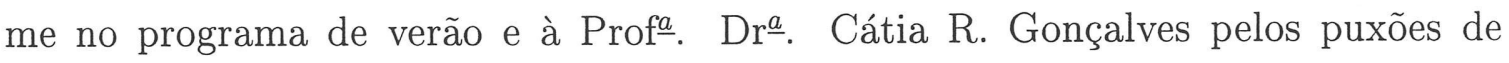
orelha. Também agradeço ao Heraldo S. Zangirolami e ao Marcos R. P. de Souza, pela atenção e pelo carinho que sempre se dispuseram para comigo.

Aos meu queridos pais, que muitas vezes se sacrificaram para me verem chegar aqui.

Ao meu amado Júnior, que mesmo estando longe durante todo esse tempo, me apoiou dando exemplo de luta e coragem.

Às minhas "irmãzinhas": Luciene (Simplesmente pelo que você é!), Andréa (Pelas palavras sábias!) e Daniela (Pelo carinho e paciência!). À Luciana pela doçura e presteza. Aos amigos: Miguel, Vera, Maria Alice, Rubinha, José, Lee, Romário, Claudemir, Daniel, Eliane, Márcio e Maria Trombini.

E ainda, aos funcionários do ICMC e a todos aqueles que de uma forma ou de outra contribuíram para a realização desta dissertação.

Que Deus abençoe todos vocês! 


\section{Sumário}

Introdução

1 Preliminares 5

1.1 Campos Vetoriais . . . . . . . . . . . . . . . 5 5

1.2 Métricas definidas por campos vetoriais . . . . . . . . . . . 7

1.3 Volumes de bolas . . . . . . . . . . . . . . . . . . . . . . 11

1.4 Outros conceitos . . . . . . . . . . . . . . . . . 14

2 As classes dos pesos $A_{p}, A_{\infty}$ e $A_{\infty}$-forte $\quad 15$

2.1 A classe dos pesos $A_{p} \ldots \ldots \ldots \ldots \ldots$

2.1 .1 Alguns resultados . . . . . . . . . . . . . . 16

2.2 A classe dos pesos $A_{\infty} \ldots \ldots \ldots \ldots . \ldots . \ldots . \ldots . \ldots 24$

2.3 A classe dos pesos $A_{\infty}$-forte . . . . . . . . . . . . . . 32

3 Desigualdades de Poincaré e Fórmulas de Representação 39

3.1 Desigualdade de Poincaré usual . . . . . . . . . . . . . . . 40

3.2 Condições necessárias para a existência de fórmulas de representação 41

3.3 Condições necessárias para desigualdades do tipo de Poincaré . . . . . 47

4 Aplicações $\quad 49$

4.1 Equivalência da desigualdade de Poincaré e existência de fórmulas de representação para campos vetoriais de Hörmander . . . . . . . . . . 49

4.2 Equivalência da desigualdade de Poincaré e existência de fórmulas de representação para campos vetoriais de Grushin . . . . . . . . . . 56

$\begin{array}{lc}\text { Referências Bibliográficas } & 61\end{array}$ 


\section{Abstract}

In this work we studied the relationship between $L^{1}$ versions of weighted Foincaré's inequalities and the existence of representation formulas for first order vector fields. 


\section{Introdução}

Para uma função $f \in \operatorname{Lip}(\bar{B})$, (espaço das funções lipschitzianas em $\bar{B}$ ), onde $B$ é uma bola no $\mathbb{R}^{N}$, temos que

$$
\left|f(x)-f_{B}\right| \leq C \int_{B} \frac{|\nabla f(y)|}{|x-y|^{N-1}} d y
$$

para todo $x \in B$, onde $\nabla f$ denota o gradiente de $f, f_{B}$ é a média $\frac{1}{|B|} \int_{B} f(y) d y,|B|$ é a medida de Lebesgue de $B$ e $C$ é uma constante independente de $f, x$ e $B$. Esta desigualdade é uma fórmula de representação envolvendo o campo gradiente de $f$.

Seja $\Omega \subset \mathbb{R}^{N}$ um aberto e conexo e sejam $X_{1}, \ldots, X_{p}$ campos vetoriais definidos em uma vizinhança de $\bar{\Omega}$ satisfazendo a condição de Hörmander de ordem $M$ para algum inteiro $M$, ou seja, existe um inteiro $M$ tal que os campos vetoriais $X_{1}, \ldots, X_{p}$ juntamente com seus comutadores de comprimento menor ou igual a $M$ geram o $\mathbb{R}^{N}$ em cada $x \in \bar{\Omega}$. Para estes campos vetoriais é possível associar naturalmente uma métrica $\rho$ e definir uma família de bolas $B=B(x, \rho(x, y))$ a partir desta métrica, e sob certas condições, obter uma fórmula de representação do tipo

$$
\left|f(x)-f_{B}\right| \leq \int_{\tau B}|X f(y)| \frac{\rho(x, y)}{\mid B(x, \rho(x, y) \mid} d y,
$$

para todo $x \in B$ e $\tau>1$, onde $\tau B$ é a bola concêntrica com $B$ e raio $\operatorname{\tau r}(B)$ se $r(B)$ é o raio de $B$. Veremos que se $\nu$ e $\mu$ forem medidas dobrantes em um espaço homogêneo $(\mathcal{S}, \rho, \mu)$, (ver Definição 1.25), satisfazendo as condições

$$
\frac{r(B)}{\mu(B)} \leq\left(\frac{r(\widetilde{B})}{r(B)}\right)^{\epsilon} \frac{r(\widetilde{B})}{\mu(\widetilde{B})}
$$

para todas as bolas $B$ e $\widetilde{B}$ tais que $\widetilde{B} \subset B \subset \tau k B_{0}$ e

$$
\frac{1}{\nu(B)} \int_{B}\left|f-f_{B, \nu}\right| d \nu \leq C \frac{r(B)}{\mu(B)} \int_{B}|X f| d \nu,
$$

para toda bola $B \subset \tau k B_{0}$, onde $\tau>1, \epsilon>0, k$ é uma constante dependendo de $\rho, B_{0}$ é uma bola fixa em $\Omega$ definida a partir da métrica $\rho, f \in \operatorname{Lip}\left(\tau k B_{0}\right)$ e $f_{B, \nu}$ 
é média de $f$ com relação à medida $\nu$, então teremos a fórmula de representação (1) com as medidas $\nu$ e $\mu$, como pode ser visto no Teorema 3.5. A desigualdade (3) é conhecida como desigualdade do tipo de Poincaré e sob certas condições são equivalentes às fórmulas de representação, são equivalentes à (1). Por exemplo se tivermos para uma bola $B$, algum $\tau \geq 1$ e alguma constante $c_{B}$

$$
\left|f(x)-c_{B}\right| \leq C \int_{\tau B} \phi(B(x, \rho(x, y))) d \sigma(y), \quad \text { para } \nu \text {-quase todo } x \in B,
$$

com $\phi$ satisfazendo a relação

$$
\frac{1}{\nu(B)} \int_{B} \phi(B(x, \rho(x, y))) d \nu(x) \leq C \phi(B), \text { para } \sigma \text {-quase todo } y \in \tau B,
$$

teremos a desigualdade de Poincaré

$$
\frac{1}{\nu(B)} \int_{B}\left|f(x)-c_{B}\right| d \nu(x) \leq \phi(B) \sigma(B) .
$$

Num contexto mais geral, se trocarmos (2) por

$$
\phi(B) \leq C\left(\frac{r(\widetilde{B})}{r(B)}\right)^{\epsilon} \phi(\widetilde{B}), \quad \widetilde{B} \subset c_{1} B
$$

com centro de $\widetilde{B}$ em $B$ e se trocarmos (3) por

$$
\frac{1}{\nu(B)} \int_{B}\left|f-f_{B, \nu}\right| d \nu \leq \phi(B) \sigma(B)
$$

onde $\phi$ é uma função não negativa de bolas $B, \sigma$ é uma medida e $c_{1}$ é uma constante suficientemente grande dependendo somente de $\tau$ e $k$, obtemos como conclusão que para $\nu$-quase todo $x \in B_{0}$

$$
\left|f(x)-f_{B, \nu}\right| \leq C \int_{\tau k B_{0}} \phi(B(x, \rho(x, y)) d \sigma(y) .
$$

O objetivo deste trabalho é estudar os teoremas principais apresentados em [FLW1], por Franchi, Lu e Wheeden, onde são demonstradas as condições que asseguram quando desigualdades de Poincaré implicam uma fórmula de representação e vice-versa, como pode ser observado no Capítulo 3.

No Capítulo 4 apresentamos dois exemplos em que verificaremos a equivalência dos teoremas citados acima, sendo que o primeiro exemplo trata o caso em que a métrica está associada a campos vetoriais satisfazendo a condição de Hörmander de ordem $M$ e $|X f|=\sum_{i=1}^{M}\left|X_{i} f\right|$ para $d \mu=d \nu=d x$. 
No segundo exemplo é considerado o caso em que os campos vetoriais são do tipo Grushin, ou seja, são campos vetoriais da forma

$$
X_{1}=\frac{\partial}{x_{1}}, \ldots, X_{n}=\frac{\partial}{x_{n}}, X_{n+1}=\lambda(x) \frac{\partial}{y_{n+1}}, \ldots, X_{m}=\lambda(x) \frac{\partial}{y_{m}},
$$

com $n, m \geq 1, n+m=N, \lambda \in C^{\infty}\left(\mathbb{R}^{N}\right)$, e $d \mu=\omega^{1-\frac{1}{N}} d x$, onde $\omega$ é um peso $A_{\infty}$-forte, como em [FGW].

Para definir e estudar a classe dos pesos $A_{\infty}$-forte foi necessário inicialmente estudar a classe dos pesos $A_{p}$, ou seja, a classe das funções $\omega \in L_{l o c}^{1}$ satisfazendo

$$
\frac{1}{|B|} \int_{B} \omega d x\left(\frac{1}{|B|} \omega^{-\frac{p^{\prime}}{p}}\right)^{\frac{p}{p^{\prime}}} \leq C, \text { se } 1<p<\infty
$$

ou

$$
\frac{1}{|B|} \int_{B} \omega d x \leq C \operatorname{infess}_{x \in B} \omega(x), \text { se } p=1
$$

para toda bola $B \subset \mathbb{R}^{N}$. A união de todas essas classes $A_{p}, p \geq 1$ é denotada por $A_{\infty}$. Pra um peso $\omega \in A_{p}$ definiremos uma medida $d \mu=\omega d x$, teremos uma medida dobrante, e portanto, uma medida dobrante reversa (maiores detalhes serão encontrados no Capítulo 2) e com isso, pode-se provar a validade das condições (2) e (3).

No Capítulo 1 introduzimos os conceitos e notações necessários para para o andamento deste trabalho, como as definições de campos vetoriais, condição de Hörmander e campos do tipo Grushin, métricas associadas aos campos vetoriais, bolas definidas a partir desta métrica, e outros conceitos. 


\section{Capítulo 1}

\section{Preliminares}

Neste capítulo definiremos vários conceitos e apresentaremos algumas notações e resultados que serão necessários para o desenvolvimento do nosso trabalho.

Para dois números reais positivos $D_{1}$ e $D_{2}$ escreveremos $D_{1} \lesssim D_{2}$ quando existir uma constante $C$ tal que $D_{1} \leq C D_{2}$. E escreveremos $D_{1} \approx D_{2}$ quando existirem constantes $C_{1}$ e $C_{2}$ tais que $C_{1} D_{1} \leq D_{2} \leq C_{2} D_{1}$.

Em vários contextos, embora usemos a mesma notação $C$ para as constantes que aparecem nas desigualdades, elas podem não serem as mesmas quando mudamos de uma linha para outra.

\subsection{Campos Vetoriais}

Iniciaremos com a definição de campo vetorial, ferramentas de grande utilidade neste trabalho.

Definição 1.1. Seja $\mathcal{A}$ uma álgebra sobre um corpo $K$. Uma derivação de $\mathcal{A}$ é uma aplicação $D: \mathcal{A} \rightarrow \mathcal{A}$ tal que

(i) $D(\alpha f+\beta g)=\alpha D f+\beta D g, \quad \alpha, \beta \in K$ ef,g $\in \mathcal{A}$;

(ii) $D(f g)=f(D g)+(D f) g, \quad f, g \in \mathcal{A}$.

Definição 1.2. Um campo vetorial $X$ sobre uma variedade $V$ de classe $C^{\infty}$ é uma derivação da álgebra $C^{\infty}(V)$. 
Seja $\mathcal{D}^{1}(V)$ o conjunto de todos os campos vetoriais sobre $V$. Se $f \in C^{\infty}(V)$ e $X, Y$ são campos vetoriais sobre $\mathrm{V}$, então $f X$ e $X+Y$ denotam os campos vetoriais

$$
\begin{array}{ccrl}
f X: g & \mapsto f(X g), & g \in C^{\infty}(V), \\
X+Y: g & \mapsto X g+Y g, & g \in C^{\infty}(V) .
\end{array}
$$

Se $X, Y$ são campos vetoriais sobre $\mathrm{V}$, então $X Y-Y X$ é também uma derivação de $C^{\infty}(V)$ e é denotado por $[X, Y]$, e nós referimos a $[X, Y]$ como sendo o comutador de $X$ por $Y$.

O comutador satisfaz a identidade de Jacobi

$$
[X,[Y, Z]]+[Y,[Z, X]]+[Z,[X, Y]]=0 .
$$

Observação 1.3. É imediato por (ii) da Definição 1.2 que se $f$ é uma função constante e $X \in \mathcal{D}^{1}$, então $X f=0$.

Consideremos o lema a seguir, que está provado em $[\mathrm{H}]$ às páginas 6 e 7 .

Lema 1.4. Seja $K$ um subconjunto compacto de uma variedade $V$ e seja $\Omega$ um subconjunto aberto de $V$ contendo $K$. Então existe uma função $\psi \in C^{\infty}(V)$ que é identicamente 1 em $K$ e identicamente 0 fora de $\Omega$.

Suponhamos agora, que uma função $g \in C^{\infty}(V)$ se anula sobre um subconjunto aberto $\Omega \subset V$. Seja $x$ um ponto arbitrário em $\Omega$. De acordo com o Lema 1.4, existe uma função $f \in C^{\infty}(V)$ tal que $f(x)=0$ e $f=1$ fora de $\Omega$. Então $g=f g$ e também,

$$
X g=f(X g)+g(X f)
$$

o que mostra que $X g$ se anula em $x$. Como $x$ foi tomado arbitrário, $X g=0$ sobre $\Omega$.

Assim, podemos definir $X f$ sobre $\Omega$ para cada função $f \in C^{\infty}(\Omega)$. Se $x \in \Omega$, escolhendo $\tilde{f} \in C^{\infty}(V)$ tal que $f$ e $\tilde{f}$ coincidam em uma vizinhança de $x$, temos

$$
(X f)(x)=(X \tilde{f})(x)
$$

Note que as considerações acima mostram que a definição de campo vetorial sobre $\Omega \subset V$ é válida independentemente da escolha de $\tilde{f}$.

Por todo este trabalho, estaremos considerando $V=\mathbb{R}^{N}$. 
Definição 1.5. Seja $\Omega \subset \mathbb{R}^{N}$ um conjunto aberto e conexo e sejam $X_{1}, \ldots, X_{p}$ campos vetoriais $C^{\infty}$. Dizemos que estes campos satisfazem a condição de Hörmander de ordem $M$, se existir um inteiro $M$ tal que $X_{1}, \ldots, X_{p}$ e seus comutadores

$$
\left[X_{i},\left[X_{i+1}, \ldots,\left[X_{M-1}, X_{M}\right]\right], \ldots\right]
$$

de comprimento menor ou igual a $M$ geram o $\mathbb{R}^{N}$ em cada ponto $x \in \bar{\Omega}$.

\section{Exemplos.}

1. $\frac{\partial}{\partial x_{1}}, \ldots, \frac{\partial}{\partial x_{N}}$ em $\mathbb{R}^{N}$ são campos vetoriais de Hörmander de ordem $M=1$.

2. $X_{1}=\partial_{1}+2 x_{2} \partial_{3}, X_{2}=\partial_{2}-2 x_{1} \partial_{3}, X_{3}=x_{1} \partial_{2}$ em $\mathbb{R}^{3}$ são campos vetoriais de Hörmander de ordem $M=2$, já que $\left\{X_{1}, X_{2},\left[X_{1}, X_{2}\right]\right\}$ gera o $\mathbb{R}^{3}$ em todos pontos. Note que $\left[X_{1}, X_{2}\right]=-4 \partial_{3}$.

Definição 1.6. $X_{1}, \ldots, X_{N}$ são chamados campos vetoriais de Grushin, se $X_{1}, \ldots, X_{N}$ são campos vetoriais da forma

$$
X_{1}=\frac{\partial}{\partial x_{1}}, \ldots, X_{n}=\frac{\partial}{\partial x_{n}}, X_{n+1}=\lambda(x) \frac{\partial}{\partial y_{1}}, \ldots, X_{N}=\lambda(x) \frac{\partial}{\partial y_{m}}
$$

$\operatorname{com} n, m \geq 1, n+m=N, \lambda \in C^{\infty}\left(\mathbb{R}^{n}\right)$.

Observação 1.7. Uma função suave $F: \mathbb{R}^{N} \rightarrow \mathbb{R}^{N}$ dá origem a um campo vetorial em $\mathbb{R}^{N}$ da seguinte maneira:

$$
X(f)=\langle F, \nabla f\rangle
$$

isto é,

$$
X(f)(x)=F_{1}(x) \frac{\partial}{\partial x_{1}} f(x)+\cdots+F_{1}(x) \frac{\partial}{\partial x_{N}} f(x),
$$

onde $F_{1}, \ldots, F_{N}$ são as componentes de $F$.

\subsection{Métricas definidas por campos vetoriais}

Nesta seção definiremos uma métrica $\rho$ a partir de campos vetoriais e observaremos que existem outras maneiras de definir métricas associadas a campos vetoriais, mas que são todas equivalentes, e por isso, poderemos utilizar ambas as formas, dependendo do contexto que estivermos considerando. 
Seja $\Omega \subset \mathbb{R}^{N}$ um conjunto aberto e conexo e sejam $X_{0}, X_{1}, \ldots, X_{p}$ campos vetoriais reais $C^{\infty}$ definidos em uma vizinhança de $\bar{\Omega}$. Sejam

$$
\begin{aligned}
& X^{(1)}=\left\{X_{0}, X_{1}, \ldots, X_{p}\right\} \\
& X^{(2)}=\left\{\left[X_{0}, X_{1}\right], \ldots,\left[X_{p-1}, X_{p}\right]\right\}, \text { etc. }
\end{aligned}
$$

tais que os elementos de $X^{(k)}$ sejam os comutadores de comprimento $k$. Seja $Y_{1}, \ldots, Y_{q}$ alguma enumeração dos componentes de $X^{(1)}, \ldots, X^{(M)}$. Se $Y_{i}$ é um elemento de $X^{(j)}$, dizemos que $Y_{i}$ tem grau formal deg $\left(Y_{i}\right)=j$.

Faremos ainda as seguintes hipóteses sobre os campos vetoriais:

(a) Para cada $j$ e $k$ podemos escrever

$$
\left[Y_{j}, Y_{k}\right]=\sum_{\operatorname{deg}\left(Y_{l}\right) \leq \operatorname{deg}\left(Y_{j}\right)+\operatorname{deg}\left(Y_{k}\right)} c_{j k}^{l} Y_{l}
$$

onde $c_{j k}^{l} \in C^{\infty}(\bar{\Omega})$.

(b) Para cada $x \in \bar{\Omega}$, os vetores $Y_{1}(x), \ldots, Y_{q}(x)$ geram o $\mathbb{R}^{N}$.

Um exemplo simples ocorre quando $X_{1}, \ldots, X_{q}$ são campos vetoriais satisfazendo a condição de Hörmander. De fato, a propriedade (a) segue da Identidade de Jacobi, e a propriedade (b) é imediata.

Para campos vetoriais $Y_{1}, \ldots, Y_{q}$ em $\Omega$ satisfazendo as hipóteses acima, podemos definir uma métrica em $\Omega$ como a seguir.

Definição 1.8. Uma função $f:[a, b] \subset \Omega \rightarrow \mathbb{R}^{N}$ é absolutamente contínua sobre $[a, b]$, se para cada $\epsilon>0$, existir um $\delta>0$ tal que

$$
\sum_{i=1}^{k}\left|f\left(y_{i}\right)-f\left(x_{i}\right)\right|<\epsilon
$$

para cada coleção disjunta e finita $\left\{\left(x_{i}, y_{i}\right): i=1,2, \ldots, k\right\}$ de intervalos em $[a, b]$, com

$$
\sum_{i=1}^{k}\left|y_{i}-x_{i}\right|<\delta .
$$

Definição 1.9. Para cada $\delta>0$, denotemos por $C(\delta)$ a classe das aplicações absolutamente contínuas $\varphi:[0,1] \rightarrow \Omega$ as quais satisfazem a equação diferencial

$$
\varphi^{\prime}(t)=\sum_{j=1}^{q} a_{j}(t) Y_{j} \varphi(t),
$$


$\operatorname{com}\left|a_{j}(t)\right|<\delta^{\operatorname{deg}\left(Y_{j}\right)}$. Nestas condições então definimos

$$
\rho(x, y)=\inf \{\delta>0: \exists \varphi \in C(\delta) \operatorname{com} \varphi(0)=x \text { e } \varphi(1)=y\}
$$

Uma outra maneira de definir uma métrica pode ser feita considerando-se $\gamma$ : $[0, T] \rightarrow \Omega$ uma curva sub-unitária, ou seja, uma curva absolutamente contínua satisfazendo a condição

$$
\left|\left\langle\gamma^{\prime}(t), \xi\right\rangle\right|^{2} \leq \sum_{j}\left|\left\langle X_{j}(\gamma(t)), \xi\right\rangle\right|^{2}
$$

para todo $\xi \in \mathbb{R}^{N}$ e quase todo $t \in[0, T]$. Para essa curva podemos definir, para cada $x, y \in \Omega$,

$$
\begin{gathered}
\varrho(x, y)=\inf \{T: \exists \text { uma curva sub-unitária } \gamma:[0, T] \rightarrow \Omega \\
\operatorname{com} \gamma(0)=x \text { e } \gamma(T)=y\} .
\end{gathered}
$$

Temos o seguinte resultado:

Proposição 1.11. Seja $\rho$ definida como em (1.10), e $M=\max \operatorname{deg}\left(Y_{j}\right)$. Então $\rho$ é uma métrica e se $K \subset \Omega$ é um conjunto compacto, existem constantes $c_{1}, c_{2}$ tais que se $x, y \in K$,

$$
c_{1}|x-y| \leq \rho(x, y) \leq c_{2}|x-y|^{\frac{1}{M}} .
$$

\section{Prova:}

Primeiramente mostraremos a validade de (1.12).

Seja $K \subset \Omega$ um conjunto compacto e $M=\max \operatorname{deg}\left(Y_{j}\right)$. Então existe uma constante $C$ tal que se $x, y \in K$, existe uma função absolutamente contínua

$$
\varphi:[0,1] \rightarrow \Omega \operatorname{com} \varphi(0)=x, \varphi(1)=y \text { e }\left|\varphi^{\prime}(t)\right| \leq C|\varphi(1)-\varphi(0)|=C|x-y|,
$$

para todo $t \in[0,1]$. Como os campos vetoriais $Y_{1}, \ldots, Y_{q}$ geram o $\mathbb{R}^{N}$ em cada ponto $x \in \Omega$, podemos escrever

$$
\varphi^{\prime}(t)=\sum_{j=1}^{q} b_{j}(t) Y_{j}(\varphi(t)),
$$

$\operatorname{com}\left|b_{j}(t)\right| \leq c^{\prime}\left|\varphi^{\prime}(t)\right|$. Assim, $\left|b_{j}(t)\right| \leq c^{\prime \prime}|x-y|=c^{\prime \prime}\left(|x-y|^{\frac{1}{\operatorname{deg}\left(Y_{j}\right)}}\right) \operatorname{deg}\left(Y_{j}\right)$, para todo $j=1, \ldots, q$. 
Se $|x-y|<1$, então como $\operatorname{deg}\left(Y_{j}\right) \leq M,|x-y|^{\frac{1}{\operatorname{deg}\left(Y_{j}\right)}} \leq|x-y|^{\frac{1}{M}}$ e portanto tomando $\delta=|x-y|^{\frac{1}{M}}$, obtemos $\left|b_{j}(t)\right| \leq c^{\prime \prime}\left(|x-y|^{\frac{1}{\operatorname{deg}\left(Y_{j}\right)}}\right)^{\operatorname{deg}\left(Y_{j}\right)} \leq c^{\prime \prime}\left(|x-y|^{\frac{1}{M}}\right)^{\operatorname{deg}\left(Y_{j}\right)}=$ $c^{\prime \prime} \delta^{\operatorname{deg}\left(Y_{j}\right)}$, e assim,

$$
\rho(x, y) \leq C|x-y|^{\frac{1}{M}}
$$

Se $|x-y| \geq 1$, então considerando $c_{0}=\operatorname{diam}(K)$,

$$
\left|b_{j}(t)\right| \leq c^{\prime \prime} 2 c_{0}\left(\left|\frac{x-y}{2 c_{0}}\right|^{\frac{1}{\operatorname{deg}\left(Y_{j}\right)}}\right)^{\operatorname{deg}\left(Y_{j}\right)}=C_{2}\left(\left|x_{2}-y_{2}\right|^{\frac{1}{\operatorname{deg}\left(Y_{j}\right)}}\right)^{\operatorname{deg}\left(Y_{j}\right)} .
$$

onde $x_{2}=\frac{x}{2 c_{0}}$ e $y_{2}=\frac{y}{2 c_{0}}$. E o resultado segue analogamente, já que $\left|x_{2}-y_{2}\right|<1$.

Por outro lado, se $x, y \in K$ e $\rho(x, y)=\delta$, existe $\varphi \in C(2 \delta) \operatorname{com} \varphi(0)=x$, $\varphi(1)=y$ e $\varphi^{\prime}(t)=\sum_{j=1}^{q} a_{j}(t) Y_{j}(\varphi(t))$ com $\left|a_{j}(t)\right| \leq(2 \delta)^{\operatorname{deg}\left(Y_{j}\right)}$. Como as componentes de cada $Y_{j}$ são uniformemente limitadas, pois são de classe $C^{\infty}(\bar{\Omega})$ e $\bar{\Omega}$ é compacto, segue que

$$
\left|\varphi^{\prime}(t)\right| \leq \sum_{j=1}^{q}\left|a_{j}(t) Y_{j}(\varphi(t))\right| \leq C \sum_{j=1}^{q}(2 \delta)^{\operatorname{deg}\left(Y_{j}\right)} \leq C^{\prime} \delta
$$

para $\delta<1$. Se $\delta \geq 1$, então $|x-y| \leq c_{0} \leq c_{0} \delta$ e também temos $\left|\varphi^{\prime}(t)\right| \leq C \delta$. Portanto

$$
|x-y|=\left|\int_{0}^{1} \varphi^{\prime}(t) d t\right| \leq C^{\prime} \delta
$$

e (1.12) está provada.

Agora se $\rho(x, y)=0$, então por (1.12), teremos $x=y$ e portanto a primeira condição de métrica está verificada. As demais propriedades seguem imediatamente da definição.

Segue por esta proposição que a métrica $\rho: \Omega \times \Omega \rightarrow[0, \infty)$ é contínua com relação a Topologia Euclidiana, e podemos então definir uma família de bolas $B(x, \delta)$ sobre $\Omega$ por

$$
B(x, \delta)=\{y \in \Omega: \rho(x, y)<\delta\}
$$

E temos o seguinte resultado:

Corolário 1.14. As bolas definidas em (1.13) satisfazem as seguintes propriedades:

(i) $B(x, \delta)$ é aberta e se $\delta>0, B(x, \delta)=\cup_{s<\delta} B(x, s)$; 
(ii) $\cap_{s>0} B(x, s)=\{x\}$;

(iii) Para cada conjunto compacto $K \subset \Omega$, existe uma constante positiva $C$ tal que se $x, y \in K, 0<\delta_{1}<\delta_{2}$ e $B\left(x, \delta_{1}\right) \cap B\left(y, \delta_{2}\right) \neq \varnothing$, então $B\left(x, \delta_{1}\right) \subset B\left(y, C \delta_{2}\right)$.

Prova: Basta lembrar que $\rho$ é uma métrica.

Agora vamos introduzir uma noção de equivalência para famílias de bolas.

Definição 1.15. Dizemos que duas funções $\rho_{1}, \rho_{2}: \Omega \times \Omega \rightarrow[0, \infty]$ são equivalentes se para cada compacto $K \subset \Omega$ existir uma constante $C$ tal que se $x, y \in K$,

$$
\frac{1}{C} \rho_{2}(x, y) \leq \rho_{1}(x, y) \leq C \rho_{2}(x, y)
$$

Dizemos que $\rho_{1}$ e $\rho_{2}$ são localmente equivalentes se para cada $x_{0} \in \Omega$ existe uma vizinhança aberta $U$ contendo $x_{0}$ tal que $\rho_{1}$ e $\rho_{2}$ são equivalentes em $U$.

Se $\rho_{1}$ e $\rho_{2}$ são quasi-distâncias e $B_{1}, B_{2}$ são respectivamente bolas das famílias correspondentes a $\rho_{1}$ e $\rho_{2}$, então a equivalência ocorre exatamente quando para cada compacto $K \subset \Omega$ existir uma constante $C$ tal que para todo $x \in K$,

$$
B_{1}(x, \delta) \subset B_{2}(x, C \delta) \text { e } B_{2}(x, \delta) \subset B_{1}(x, C \delta) .
$$

\subsection{Volumes de bolas}

Utilizaremos a notação $|B|$ para indicar a medida de Lebesgue de $B$.

Como vimos na seção 1.2, a função $\rho$ é uma métrica e sua família de bolas correspondente $B(x, \delta)$ satisfaz as condições $(i),(i i)$ e $(i i i)$ do Corolário 1.14. Um dos maiores objetivos desta seção é mostrar que a métrica $\rho$ também satisfaz a propriedade dobrante para volume, ou seja, queremos provar o seguinte resultado:

Proposição 1.16. Para cada compacto $K \subset \Omega$, existe uma constante positiva $C$ tal que se $x \in K$ e $\delta>0$, então

$$
|B(x, 2 \delta)| \leq C|B(x, \delta)|
$$


Para provar esta proposição, necessitaremos de várias estimativas para o volume das bolas $B(x, \delta)$, como a seguir.

Para cada $N$-upla de inteiros $I=\left(i_{1}, \ldots, i_{N}\right)$ com $1 \leq i_{j} \leq q$, defina

$$
\lambda_{I}(x)=\operatorname{det}\left(Y_{i_{1}}, \ldots, Y_{i_{N}}\right)(x) .
$$

Considere também

$$
d(I)=\operatorname{deg}\left(Y_{i_{1}}\right)+\cdots+\operatorname{deg}\left(Y_{i_{N}}\right) .
$$

E então defina

$$
\Lambda(x, \delta)=\sum_{I}\left|\lambda_{I}(x)\right| \delta^{d(I)}
$$

onde a soma é feita sobre todas as $N$-uplas $I$. Com esta notação podemos apresentar um resultado importante sobre o volume de bolas $B(x, \delta)$, o qual está provado em [NSW] à página 126.

Teorema 1.18. Para cada conjunto compacto $K \subset \Omega \subset \mathbb{R}^{N}$, existem constantes $c_{1}$ e $c_{2}$ tais que para todo $x \in K$ e $\delta>0$, tem-se

$$
0<c_{1} \Lambda(x, \delta) \leq|B(x, \delta)| \leq c_{2} \Lambda(x, \delta)<\infty
$$

Prova da Proposição 1.16: Pelo Teorema 1.18 obtemos

$$
c_{1} \Lambda(x, 2 \delta) \leq|B(x, 2 \delta)| \leq c_{2} \Lambda(x, 2 \delta)
$$

para todo $x \in K$. Mas, por (1.17), podemos escrever

$$
\Lambda(x, 2 \delta)=\sum_{I}\left|\lambda_{I}(x)\right|(2 \delta)^{d(I)} \leq 2^{N M} \sum_{I}\left|\lambda_{I}(x)\right| \delta^{d(I)}=2^{N M} \Lambda(x, \delta) .
$$

Portanto, da segunda desigualdade em (1.19) e pelo mesmo teorema, temos

$$
|B(x, 2 \delta)| \leq c_{2} 2^{N M} \Lambda(x, \delta) \leq \frac{c_{2} 2^{N M}}{c_{1}}|B(x, \delta)|=C|B(x, \delta)|,
$$

ou seja, $\rho$ satisfaz a propriedade dobrante, e a Proposição 1.16 está provada.

E como conseqüência do Teorema 1.18, temos o seguinte resultado: 
Corolário 1.20. Para cada conjunto compacto $K \subset \Omega \subset \mathbb{R}^{N}$, existem constantes $c_{1}$ e $c_{2}$ tais que se $x \in K$ e $0 \leq s<r$, tem-se

$$
c_{1}\left(\frac{r}{s}\right)^{\alpha} \leq \frac{|B(x, r)|}{|B(x, s)|} \leq c_{2}\left(\frac{r}{s}\right)^{\beta}
$$

para $\alpha=\alpha(x)$ e $\beta=\beta(x)$ convenientes $\operatorname{com} N \leq \alpha \leq \beta$.

Prova: Para provar (1.21), primeiro lembremos que, pela condição de Hörmander, existe um inteiro positivo $M$ tal que dentre $X_{1}, \ldots, X_{p}$ e seus comutadores de grau menor ou igual a $M$, podemos encontrar uma $N$-upla de campos vetoriais que são linearmente independentes em cada $x \in K$. Agora defina

$$
\begin{aligned}
& \alpha=\alpha(x)=\min \left\{\operatorname{deg} Y_{i_{1}}+\cdots+\operatorname{deg} Y_{i_{N}}\right\} \\
& \beta=\beta(x)=\max \left\{\operatorname{deg} Y_{i_{1}}+\cdots+\operatorname{deg} Y_{i_{N}}\right\},
\end{aligned}
$$

onde $\operatorname{deg} Y_{i}$ é o grau formal de $Y_{i}$ (um inteiro fixo $\geq 1$ ) e $Y_{i_{1}}, \ldots, Y_{i_{N}}$ cobre todas as coleções de $\mathrm{N}$ vetores escolhidos a partir de $\left\{X_{j}\right\}_{j=1}^{p}$ e seus comutadores de grau menor ou igual a $M$ tais que $Y_{i_{1}}, \ldots, Y_{i_{N}}$ sejam linearmente independentes em $x$.

Claramente $\alpha \geq N$ e $\beta \leq M N$.

Agora, pelo Teorema 1.18, se $x \in K$ e $0<r$, então

$$
|B(x, r)| \approx \Lambda(x, r)=\sum_{I}\left|\lambda_{I}(x)\right| r^{d(I)}
$$

com constantes de equivalência dependendo de $K$. Como

$$
\Lambda(x, r)=\sum_{I}\left|\lambda_{I}(x)\right| r^{d(I)}=\sum_{I}\left|\lambda_{I}(x)\right| s^{d(I)}\left(\frac{r}{s}\right)^{d(I)},
$$

e estamos supondo $s<r$ e $\lambda_{I}(x) \neq 0$, temos

$$
\left(\frac{r}{s}\right)^{\alpha} \leq\left(\frac{r}{s}\right)^{d(I)} \leq\left(\frac{r}{s}\right)^{\beta}
$$

E segue que se multiplicarmos a desigualdade acima por $\left|\lambda_{I}(x)\right| s^{d(I)}$ e fizermos a soma sobre todas a $N$-uplas $I$, obtemos

$$
\sum_{I}\left(\frac{r}{s}\right)^{\alpha}\left|\lambda_{I}(x)\right| s^{d(I)} \leq \sum_{I}\left|\lambda_{I}(x)\right| r^{d(I)} \leq \sum_{I}\left(\frac{r}{s}\right)^{\beta}\left|\lambda_{I}(x)\right| s^{d(I)},
$$

ou seja,

$$
\left(\frac{r}{s}\right)^{\alpha} \Lambda(x, s) \leq \Lambda(x, r) \leq\left(\frac{r}{s}\right)^{\beta} \Lambda(x, s)
$$


E pela equivalência (1.22),

$$
c_{1}\left(\frac{r}{s}\right)^{\alpha} \leq \frac{|B(x, r)|}{|B(x, s)|} \leq c_{2}\left(\frac{r}{s}\right)^{\beta},
$$

e o corolário está provado.

\subsection{Outros conceitos}

Também precisaremos das seguintes definições:

Definição 1.23. (Função Característica) Definimos a função característica do conjunto $A \subset \Omega$ como sendo a função $\chi_{A}: \Omega \rightarrow \mathbb{R}$ tal que

$$
\chi_{A}(x)=\left\{\begin{array}{ll}
1 & \text { se } x \in A \\
0 & \text { se } x \notin A
\end{array} .\right.
$$

Definição 1.24. (Condição de Boman) $S e(\mathcal{S}, d)$ é um espaço métrico, dizemos que um conjunto aberto $D \subset \mathcal{S}$ satisfaz a condição da cadeia de Boman $\mathcal{B}(\tau, \varsigma)$, $\tau \geq 1, \varsigma \geq 1$, se existir uma cobertura $W$ de $D$ constituída de bolas $B$ tais que

(i) $\sum_{B \in W} \chi_{\tau B}(x) \leq \varsigma \chi_{D}(x)$ para todo $x \in \mathcal{S}$;

(ii) Existe uma bola "central" $B_{1} \in W$ que pode ser conectada a qualquer bola $B \in W$ por uma cadeia finita de bolas $B_{1}, \ldots, B_{l(B)}$. E além disso, $B_{j} \cup B_{j-1}$ contém uma bola $B_{j_{0}}$ tal que $B_{j} \cap B_{j-1} \subset \varsigma B_{j_{0}}$ para $j=2, \ldots, l(B)$.

Definição 1.25. (Espaço homogêneo) Dizemos que $(\mathcal{S}, \rho, \mu)$ é um espaço homogêneo se $\rho$ é uma quasi-métrica e $\mu$ é uma medida dobrante.

Lema 1.26. Se $f \geq 0$ e $f \in L^{p}(E)$, então

$$
\int_{E} f^{p} d \mu=p \int_{0}^{\infty} \alpha^{p-1} \omega(\alpha) d \alpha,
$$

onde $\omega(\alpha)=\mu(\{x \in E: f(x)>\alpha\})$. 


\section{Capítulo 2}

\section{As classes dos pesos $A_{p}, A_{\infty} \mathrm{e}$ $A_{\infty}$-forte}

O objetivo principal deste capítulo é apresentar a classe dos pesos $A_{\infty}$-forte e estudar algumas de suas propriedades que serão necessárias para a construção dos nossos exemplos no Capítulo 4. Para este propósito, inicialmente será preciso estudar as classes dos pesos $A_{p}$ e $A_{\infty}$.

Os conteúdos apresentados sobre as classes dos pesos $A_{p}$ e $A_{\infty}$ são baseados em [FZ] e [S], e os da classe dos pesos $A_{\infty}$-forte são baseados em [FGW].

\subsection{A classe dos pesos $A_{p}$}

Nesta seção introduziremos a classe dos pesos $A_{p}$ e estudaremos algumas de suas propriedades.

Definição 2.1. Sejam $1 \leq p<+\infty$ e $p^{\prime}=\frac{p}{p-1}$. Dizemos que o peso $\omega, \omega \in L_{l o c}^{1}$, $\omega \geq 0$, satisfaz a condição $A_{p}\left(\omega \in A_{p}\right)$ se existir uma constante positiva $C$ tal que para toda bola Euclidiana $B \subset \mathbb{R}^{N}$,

$$
\frac{1}{|B|} \int_{B} \omega\left(\frac{1}{|B|} \int_{B} \omega^{-\frac{p^{\prime}}{p}}\right)^{\frac{p}{p^{\prime}}} \leq C, \text { se } 1<p<\infty,
$$

ou,

$$
\frac{1}{|B|} \int_{B} \omega \leq C \text { infess }_{x \in B} \omega(x), \text { se } p=1 .
$$

Para evitar trivialidades assumiremos que $\omega$ é não nulo.

Por todo este trabalho, usaremos a notação $f_{B}$ para $\frac{1}{|B|} \int_{B} f$. 


\subsubsection{Alguns resultados}

Faremos algumas observações sobre a classe $A_{p}$.

2.1.1.1 Observe que as desigualdades opostas a (2.2) e (2.3) são válidas para qualquer peso $\omega$, onde se pode tomar $C=1$. Vejamos o caso $1<p<\infty$. Temos

$$
\begin{aligned}
|B|=\int_{B} 1=\int_{B} \omega^{\frac{1}{p}} \omega^{-\frac{1}{p}} & \leq\left(\int_{B}\left(\omega^{\frac{1}{p}}\right)^{p}\right)^{\frac{1}{p}}\left(\int_{B}\left(\omega^{-\frac{1}{p}}\right)^{\frac{p}{p-1}}\right)^{\frac{p-1}{p}} \\
& =\left(\int_{B} \omega\right)^{\frac{1}{p}}\left(\omega^{-\frac{1}{p-1}}\right)^{\frac{p-1}{p}}
\end{aligned}
$$

pela desigualdade de Hölder. Logo,

$$
1 \leq\left(\frac{1}{|B|} \int_{B} \omega\right)\left(\frac{1}{|B|} \int_{B} \omega^{-\frac{1}{p-1}}\right)^{p-1}
$$

Quando $p=1$, temos

$$
\operatorname{infess}_{x \in B} \omega(x)=\frac{1}{|B|} \int_{B} \operatorname{infess}_{x \in B} \omega(x) d y \leq \frac{1}{|B|} \int_{B} \omega .
$$

Segue da definição das classes $A_{p}$, para $1<p<\infty$, que $\omega \in A_{p}$ se, e somente se, $\omega^{\frac{-1}{p-1}} \in A_{p^{\prime}}$, onde $p^{\prime}=\frac{p}{p-1}$.

2.1.1.2 Note que o peso $\omega(x)=1, x \in \mathbb{R}^{N}$, satisfaz a condição $A_{p}$ para todo $p$ em $[1, \infty)$. Mais geralmente, $\omega(x)=|x|^{\alpha}, x \in \mathbb{R}^{N}$, satisfaz a condição $A_{p}$ se, e somente se, $-N<\alpha<N(p-1)$. Para verificar esse fato, precisaremos do seguinte resultado:

Lema 2.4. Dado $\alpha>-N$, existe uma constante positiva $C$ tal que

$$
\frac{1}{C}\left(\left|x_{0}\right|+r\right)^{\alpha} r^{N} \leq \int_{B\left(x_{0}, r\right)}|x|^{\alpha} d x \leq C\left(\left|x_{0}\right|+r\right)^{\alpha} r^{N},
$$

isto é,

$$
\left(\left|x_{0}\right|+r\right)^{\alpha} r^{N} \approx \int_{B\left(x_{0}, r\right)}|x|^{\alpha} d x
$$

para toda bola $B\left(x_{0}, r\right)$ centrada em $x_{0}$ e raio $r>0$.

Prova: Suponhamos que $2 r<\left|x_{0}\right|$. Se $x \in B\left(x_{0}, r\right)$, então

$$
|x| \leq\left|x-x_{0}\right|+\left|x_{0}\right| \leq r+\left|x_{0}\right| \leq \frac{\left|x_{0}\right|}{2}+\left|x_{0}\right|=\frac{3}{2}\left|x_{0}\right| .
$$

Também,

$$
\left|x_{0}\right| \leq\left|x-x_{0}\right|+|x| \leq r+|x| \leq \frac{\left|x_{0}\right|}{2}+|x|,
$$


e portanto,

$$
\left|x_{0}\right| \leq \frac{\left|x_{0}\right|}{2}+\frac{3}{2}\left|x_{0}\right|=2|x|
$$

Note que $\left|x_{0}\right| \approx\left|x_{0}\right|+r$, pois

$$
\left|x_{0}\right| \leq\left|x_{0}\right|+r \leq\left|x_{0}\right|+\frac{\left|x_{0}\right|}{2}=\frac{3}{2}\left|x_{0}\right|
$$

Assim,

$$
\int_{B\left(x_{0}, r\right)}\left|x_{0}\right|^{\alpha} d x \approx\left|x_{0}\right|^{\alpha} \int_{B\left(x_{0}, r\right)} d x=C_{N}\left|x_{0}\right|^{\alpha} r^{N} \approx\left(\left|x_{0}\right|+r\right)^{\alpha} r^{N}
$$

Suponhamos agora, que $2 r \geq\left|x_{0}\right|$. Se $x \in B\left(x_{0}, r\right)$, então

$$
|x| \leq\left|x-x_{0}\right|+\left|x_{0}\right| \leq 3 r
$$

isto é,

$$
B\left(x_{0}, r\right) \subset B(0,3 r)
$$

Também, $r \leq\left|x_{0}\right|+r \leq 2 r+r=3 r$, ou seja, $r \approx\left|x_{0}\right|+r$. Logo, como $\alpha>-N$,

$$
\int_{B\left(x_{0}, r\right)}|x|^{\alpha} d x \leq \int_{B(0,3 r)}|x|^{\alpha} d x=C_{N, \alpha} r^{N+\alpha} \approx\left(\left|x_{0}\right|+r\right)^{\alpha} r^{N} .
$$

Seja $y_{0}=x_{0}+\frac{x_{0}}{\left|x_{0}\right|}$ caso $x_{0} \neq 0$, ou consideremos $\left|y_{0}\right|=r$ se $x_{0}=0$. Note que $\left|y_{0}\right|=\left|x_{0}\right|+r$, o que é obvio se $x_{0}=0$, e se $x_{0} \neq 0$, segue de

$$
\left|y_{0}\right|^{2}=r^{2}+\left|x_{0}\right|^{2}+2 r\left|x_{0}\right|=\left(\left|x_{0}\right|+r\right)^{2} \text {. }
$$

Seja $B_{0}=B\left(x_{0}+\left(y_{0}-x_{0}\right) \frac{3}{4}, \frac{r}{4}\right)$. Se $x \in B_{0}$, temos

$$
\left|x-x_{0}\right| \leq\left|x-x_{0}-\left(y_{0}-x_{0}\right) \frac{3}{4}\right|+\left|x_{0}+\left(y_{0}-x_{0}\right) \frac{3}{4}-y_{0}\right| \leq \frac{r}{4}+\frac{r}{4}=\frac{r}{2},
$$

ou seja, $B \subset B\left(x_{0}, \frac{r}{2}\right) \subset B\left(x_{0}, r\right)$. Deste modo,

$$
\left|x_{0}\right|+\frac{r}{2}=\left|y_{0}\right|-\frac{r}{2} \leq|x|+\left|x-y_{0}\right|-\frac{r}{2} \leq|x| \leq\left|x-x_{0}\right|+\left|x_{0}\right| \leq r+\left|x_{0}\right|,
$$

isto é, $\left|x_{0}\right|+r \approx|x|$, se $x \in B_{0}$. Conseqüentemente,

$$
\int_{B\left(x_{0}, r\right)}|x|^{\alpha} d x \geq \int_{B_{0}}|x|^{\alpha} d x \approx\left(\left|x_{0}\right|+r\right)^{\alpha} \int_{B_{0}} d x \approx\left(\left|x_{0}\right|+r\right)^{\alpha} r^{N} .
$$

E portanto,

$$
\int_{B\left(x_{0}, r\right)}|x|^{\alpha} d x \approx\left(\left|x_{0}\right|+r\right)^{\alpha} r^{N}
$$


e isso termina a demonstração.

Provemos agora que $|x|^{\alpha}$ pertence a $A_{p}$ se, e somente se, $-N<\alpha<N(p-1)$. Suponhamos que $-N<\alpha<N(p-1), 1<p<\infty$ e que $B$ é uma bola de centro $x_{B}$ e raio $r(B)$ contida no $\mathbb{R}^{N}$. Pelo lema anterior temos

$$
\begin{aligned}
\frac{1}{|B|} \int_{B}|x|^{\alpha} d x & \left(\frac{1}{|B|} \int_{B}\left(|x|^{-\alpha}\right)^{\frac{1}{p-1}} d x\right)^{p-1} \\
& \approx \frac{C}{r(B)^{N}}\left(\left|x_{B}\right|+r(B)\right)^{\alpha} r(B)^{N}\left(\frac{1}{r(B)^{N}}\left(\left|x_{B}\right|+r(B)\right)^{-\frac{\alpha}{p-1}} r(B)^{N}\right)^{p-1} \\
& =C\left(\left|x_{B}\right|+r(B)\right)^{\alpha}\left(\left(\left|x_{B}\right|+r(B)\right)^{-\frac{-\alpha}{p-1}}\right)^{p-1} \\
& =C
\end{aligned}
$$

isto é, $|x|^{\alpha} \in A_{p}$, se $1<p<\infty$.

Reciprocamente, se $|x|^{\alpha} \in A_{p}, 1<p<\infty$, como $|x|^{\alpha}$ precisa ser localmente integrável, integrando-a sobre uma bola centrada na origem, vê-se facilmente que $-N<\alpha$. Por outro lado, como $|x|^{\alpha}$ está em $A_{p}$, existe uma constante $C_{0}$ tal que para qualquer bola centrada na origem, temos

$$
\begin{aligned}
C_{0} & \geq \frac{1}{|B|} \int_{B}|x|^{\alpha} d x\left(\frac{1}{|B|} \int_{B}|x|^{-\frac{\alpha}{p-1}} d x\right)^{p-1} \\
& \geq C_{N, \alpha} \frac{1}{|B|}|B|^{\alpha+N} \frac{1}{|B|^{p-1}}\left(\int_{B}|x|^{-\frac{\alpha}{p-1}} d x\right)^{p-1} \\
& =C_{N, \alpha}|B|^{\alpha+N-p}\left(\int_{B}|x|^{-\frac{\alpha}{p-1}} d x\right)^{p-1} .
\end{aligned}
$$

Assim, $|x|^{-\frac{\alpha}{p-1}}$ é localmente integrável e, portanto devemos ter $\frac{-\alpha}{p-1}>-N$, ou seja, $\alpha<N(p-1)$. O caso $p=1$ segue analogamente.

2.1.1.3 Existe outro modo de definir a classe $A_{p}$, o qual pode ser considerado como a seguir. Usaremos a notação $\omega(B)$ para $\int_{B} \omega(x) d x$. Para qualquer função $f$ localmente integrável e qualquer bola $\mathrm{B}$, temos a seguinte caracterização de $A_{p}$ : o peso $\omega \in A_{p}$ exatamente quando $\left(f_{B}\right)^{p}$ for limitada pelo valor médio de $f^{p}$ tomado com relação à medida $\omega(x) d x$. Mais precisamente, $\omega \in A_{p}$ se, e somente se,

$$
\left(f_{B}\right)^{p} \leq \frac{C}{\omega(B)} \int_{B} f^{p} \omega d x
$$

for válida para toda função não negativa $f$ e toda bola $B$. 
Note primeiro que (2.5) segue da desigualdade

$$
\int_{\mathbb{R}^{N}}[M f(x)]^{p} d \mu(x) \leq A \int_{\mathbb{R}^{N}}|f(x)|^{p} d \mu(x),
$$

com $d \mu=\omega(x) d x$ para todo $p, 1<p<\infty$, onde $M$ denota o operador maximal no $\mathbb{R}^{N}$ definido por

$$
(M f)(x)=\sup _{r>0} \frac{C_{N}}{r^{N}} \int_{|y| \leq r}|f(x-y)| d y,
$$

se usarmos o fato que $M f(x) \geq C^{\prime} f_{B}$, quando $x \in B$ (ver Cap. 1 de [S]). Muckenhoupt provou que (2.6) vale se, e somente se $d \mu=\omega d x$, com $\omega \in A_{p}$.

Para provarmos que (2.5) é equivalente a (2.2), assuma primeiro que $\omega \in A_{p}$. Temos

$$
f_{B}=\frac{1}{|B|} \int_{B} f(x) d x=\frac{1}{|B|} \int_{B} f(x) \omega(x)^{\frac{1}{p}} \omega(x)^{-\frac{1}{p}} d x
$$

Aplicando a desigualdade de Hölder, e utilizando (2.2), temos

$$
\begin{aligned}
\left(f_{B}\right)^{p} & =\frac{1}{|B|^{p}}\left(\int_{B} f \omega^{\frac{1}{p}} \omega^{-\frac{1}{p}}\right)^{p} \\
& \leq \frac{1}{|B|^{p}} \int_{B} f^{p} \omega\left(\int_{B} \omega^{-\frac{p^{\prime}}{p}}\right)^{\frac{p}{p^{\prime}}} \\
& =\frac{1}{|B|^{p}} \int_{B} f^{p} \omega|B|^{\frac{p}{p^{\prime}}}\left(\frac{1}{|B|} \int_{B} \omega^{-\frac{p^{\prime}}{p}}\right)^{\frac{p}{p^{\prime}}} \\
& \leq\left(C \frac{1}{|B|} \int_{B} \omega\right)^{-1} \frac{1}{|B|} \int_{B} f^{p} \omega \\
& =C \frac{1}{|B|} \int_{B} f^{p}|B| \omega(B)^{-1} \\
& =C \frac{1}{\omega(B)} \int_{B} f^{p} \omega .
\end{aligned}
$$

Portanto (2.5) está provada.

Reciprocamente, se (2.5) estiver satisfeita, então para $f=\omega^{\frac{-p^{\prime}}{p}}$ temos

$$
f^{p} \omega=\omega^{\frac{-p^{\prime}}{p} p} \omega=\omega^{1-p^{\prime}}=\omega^{\frac{-p^{\prime}}{p}}
$$

Assim, se $\int_{B} \omega^{\frac{-p^{\prime}}{p}}$ fosse finita, teríamos estabelecido (2.2). Se isso não ocorrer, trocaremos $f$ por $(\omega+\varepsilon)^{\frac{-p^{\prime}}{p}}$, onde $\varepsilon>0$ e obteremos (2.2) fazendo $\epsilon \rightarrow \infty$. De fato, 
como

$$
\begin{aligned}
\int_{B}(\omega+\epsilon)^{\frac{-p^{\prime}}{p}} d x= & \int_{B}(\omega+\epsilon)^{1-p^{\prime}} d x=\int_{B}(\omega+\epsilon)^{-p^{\prime}}(\omega+\epsilon) d x \\
& \leq \int_{B}(\omega+\varepsilon)^{-p^{\prime}} \omega d x \quad \text { pois } \epsilon>0 \\
& \leq \varepsilon^{-p^{\prime}} \int_{B} \omega d x \quad \text { pois }(\omega+\epsilon)^{-p^{\prime}} \leq \epsilon^{-p^{\prime}} \text { já que }-p^{\prime}<0 \\
& <\infty, \quad \text { pois } \omega \in L_{l o c}^{1} .
\end{aligned}
$$

Agora, usando $(2.2) \operatorname{com} f=(\omega+\epsilon)^{-\frac{p}{p^{\prime}}}=(\omega+\epsilon)^{1-p^{\prime}}$, obtemos

$$
\left(\frac{1}{|B|} \int_{B}(\omega+\epsilon)^{-\frac{p}{p^{\prime}}} d x\right)^{p} \leq \frac{C}{\omega(B)} \int_{B}(\omega+\epsilon)^{-p^{\prime}} \omega d x
$$

onde $C$ independe de $\epsilon>0$ e $B$. Daí,

$$
\left(\frac{1}{|B|} \int_{B} \omega d x\right)\left(\frac{1}{|B|} \int_{B}(\omega+\epsilon)^{1-p^{\prime}} d x\right)^{p}\left(\frac{1}{|B|} \int_{B}(\omega+\epsilon)^{-p^{\prime}} \omega d x\right)^{-1} \leq C
$$

Fazendo $\epsilon \rightarrow 0$,

$$
\begin{aligned}
&\left(\frac{1}{|B|} \int_{B} \omega d x\right)\left(\frac{1}{|B|} \int_{B} \omega^{1-p^{\prime}} d x\right)^{p}\left(\frac{1}{|B|} \int_{B} \omega^{1-p^{\prime}} d x\right)^{-1}= \\
&=\left(\frac{1}{|B|} \int_{B} \omega d x\right)\left(\frac{1}{|B|} \int_{B} \omega^{1-p^{\prime}} d x\right)^{p-1} \\
& \leq C
\end{aligned}
$$

que é (2.5). O caso $p=1$ segue analogamente.

2.1.1.4 É uma conseqüência imediata da caracterização (2.5), que quando $\omega \in$ $A_{p}$, então $\omega(x) d x$ é uma medida dobrante, isto é,

$$
\omega\left(B_{2}\right) \leq C^{\prime} \omega\left(B_{1}\right)
$$

se $B_{1}=\{x:|x-y|<\delta\}$ e $B_{2}=\{x:|x-y|<2 \delta\}$. Para ver isto, aplique (2.5) com $B=B_{2}$ e $f=\chi_{B_{1}}$. Isso nos dá $(2.7) \operatorname{com} C^{\prime}=C 2^{N p}$. De fato, como $\omega \in A_{p}$,

$$
\left(f_{B}\right)^{p} \leq \frac{C}{\omega(B)} \int_{B} f^{p} \omega
$$

para toda $f$ não negativa e para toda bola $B$. Assim,

$$
\left(\frac{1}{\left|B_{2}\right|} \int_{B_{1}} \chi_{B_{1}}(x)\right)^{p} \leq \frac{C}{\omega\left(B_{2}\right)} \int_{B_{2}} \chi_{B_{1}}(x) \omega
$$


o que implica que

$$
\left(\frac{\left|B_{1}\right|}{\left|B_{2}\right|}\right)^{p} \leq C \frac{\omega\left(B_{1}\right)}{\omega\left(B_{2}\right)}
$$

E isso nos dá

$$
\left(\frac{\delta^{N}}{(2 \delta)^{N}}\right)^{p} \leq C \frac{\omega\left(B_{1}\right)}{\omega\left(B_{2}\right)}
$$

Logo, $\omega\left(B_{2}\right) \leq 2^{N p} C \omega\left(B_{1}\right)$. E tomando $C^{\prime}=C 2^{N p}$, temos $\omega\left(B_{2}\right) \leq C^{\prime} \omega\left(B_{1}\right)$, como queríamos.

Vale observar o interessante fato de que embora um elemento da classe $A_{p}$ seja uma medida dobrante, a recíproca não é verdadeira, pois medidas da forma $\omega(x) d x$, com $\omega(x)=|x|^{\alpha}$, são medidas dobrantes quando $-N<\alpha$, e pertencem a $A_{p}$ se, e somente se, em adição, $\alpha<N(p-1)$, como vimos em 2.1.1.2.

2.1.1.5 A propriedade dobrante de $\omega$, juntamente com a caracterização (2.5), mostra que na definição $(2.2)$ dos pesos $A_{p}$, poderíamos ter substituído a família de bolas por uma família de cubos ou outras famílias equivalentes. Assim, poderemos utilizar famílias de cubos para facilitar muitas das nossas demonstrações.

2.1.1.6 Outra característica útil dos elementos da classe $A_{p}$ é dada no teorema a seguir.

Teorema 2.8. Se $1 \leq p \leq q<\infty$, então $A_{p} \subset A_{q}$.

Prova: O caso $p=q$ é trivial. Se $p=1$, então por (2.3),

$$
\text { supess } \omega^{-\frac{1}{q-1}}=(\operatorname{infess} \omega)^{-\frac{1}{q-1}} \leq\left(\frac{1}{|B|} \int_{B} \omega\right)^{-\frac{1}{q-1}} \text {. }
$$

Portanto,

$$
\frac{1}{|B|} \int_{B} \omega\left(\frac{1}{|B|} \int_{B} \omega^{-\frac{1}{q-1}}\right)^{q-1} \leq \frac{1}{|B|} \int_{B} \omega\left(\frac{1}{|B|} \int_{B} \omega\right)^{-1}=1 .
$$

Logo $\omega \in A_{q}$ para algum $q>1$.

Suponhamos então que $1<p<q<\infty$. Seja $\omega \in A_{p}$. Aplicando a desigualdade de Hölder ao par $\left(\frac{q-1}{p-1}, \frac{q-1}{q-p}\right)$, para qualquer bola $B \subset \mathbb{R}^{N}$, temos

$$
\begin{aligned}
\frac{1}{|B|} \int_{B} \omega\left(\frac{1}{|B|} \int_{B} \omega^{-\frac{1}{q-1}}\right)^{q-1} & \leq \frac{1}{|B|} \int_{B} \omega\left(\frac{1}{|B|}\left(\int_{B} \omega^{-\frac{1}{q-1}}\right)^{\frac{p-1}{q-1}}|B|^{\frac{q-p}{q-1}}\right)^{q-1} \\
& =\frac{1}{|B|} \int_{B} \omega\left(\frac{1}{|B|} \int_{B} \omega^{-\frac{1}{p-1}}\right)^{p-1} \\
& \leq C
\end{aligned}
$$


pois $\omega \in A_{p}$.

2.1.1.7 Temos também alguns resultados envolvendo a propriedade dobrante reversa, definida abaixo.

Definição 2.9. Um peso $\omega$ satisfaz a propriedade dobrante reversa, e escrevemos $\omega \in D R$, se existir $\beta>1$ tal que $\omega(2 B)>\beta \omega(B)$, para toda bola $B \subset \mathbb{R}^{N}$, onde $2 B=B(x, 2 r)$, se $B=B(x, r)$.

O próximo lema nos dá algumas propriedades relacionando pesos que satisfazem a propriedade dobrante reversa.

Lema 2.10. Temos:

(i) Se $\omega \in D R$ então existem constantes positivas $C$ e $\varepsilon$ tais que, para todas bolas concêntricas $B_{1} \subset B_{2} \subset \mathbb{R}^{N}$,

$$
\frac{\omega\left(B_{2}\right)}{\omega\left(B_{1}\right)} \geq C\left(\frac{r\left(B_{2}\right)}{r\left(B_{1}\right)}\right)^{\varepsilon},
$$

onde $r(B)$ denota o raio da bola $B$;

(ii) Se $\omega$ é dobrante então $\omega \in D R$.

Prova: Suponhamos que exista $\beta>1$ tal que $\omega(2 B)>\beta \omega(B)$ para toda bola $B \subset \mathbb{R}^{N}$. Fixe $0<r_{1}<r_{2}$ e tome $k \geq 1$ tal que

$$
2^{k} \geq \frac{r_{2}}{r_{1}}>2^{k-1}
$$

Denotemos por $B_{x}(r)$ a bola de centro $x$ e raio $r>0$ contida no $\mathbb{R}^{N}$. Observe que

$$
B_{x}\left(r_{2}\right) \supset B_{x}\left(2^{k-1} r_{1}\right),
$$

e portanto,

$$
\begin{aligned}
\omega\left(B_{x}\left(r_{2}\right)\right) & >\omega\left(B_{x}\left(2^{k-1} r_{1}\right)\right)>\beta \omega\left(B_{x}\left(2^{k-2} r_{1}\right)\right) \\
& >\beta^{2} \omega\left(B_{x}\left(2^{k-3} r_{1}\right)\right)>\beta^{k-1} \omega\left(B_{x}\left(r_{1}\right)\right) .
\end{aligned}
$$


Ou seja, podemos concluir que se $B_{1}$ e $B_{2}$ são bolas concêntricas no $\mathbb{R}^{N}$ tais que $B_{1} \subset B_{2}$, e se $\beta \geq 2$, então

$$
\begin{aligned}
\omega\left(B_{2}\right) & \geq \omega\left(B_{1}\right) \beta^{k-1}=\omega\left(B_{1}\right)\left(2^{\log _{2} \beta}\right)^{k-1} \\
& =\omega\left(B_{1}\right)\left(\beta^{k-1}\right)^{\log _{2} \beta} \geq \omega\left(B_{1}\right)\left(\frac{r_{2}}{2 r_{1}}\right)^{\log _{2} \beta} \\
& =\omega\left(B_{1}\right)\left(\frac{r_{2}}{r_{1}}\right)^{\log _{2} \beta} 2^{-\log _{2} \beta}=\omega\left(B_{1}\right) \frac{1}{\beta}\left(\frac{r_{2}}{r_{1}}\right)^{\log _{2} \beta} \\
& =C \omega\left(B_{1}\right)\left(\frac{r_{2}}{r_{1}}\right)^{\varepsilon},
\end{aligned}
$$

onde $C=\frac{1}{\beta}$ e $\varepsilon=\log _{2} \beta$. E se $\beta<2$, como $\frac{r_{2}}{r_{1}}>2^{k-1}$, então

$$
\left(\frac{r_{2}}{r_{1}}\right)^{\log _{2} \beta}<\left(2^{k-1}\right)^{\log _{2} \beta} \text {. }
$$

Assim,

$$
\omega\left(B_{2}\right)>\omega\left(B_{1}\right)\left(\frac{r_{2}}{r_{1}}\right)^{\log _{2} \beta}=C\left(\frac{r_{2}}{r_{1}}\right)^{\varepsilon} \omega\left(B_{1}\right),
$$

onde $C=1$ e $\varepsilon=\log _{2} \beta$.

Portanto,

$$
\frac{\omega\left(B_{2}\right)}{\omega\left(B_{1}\right)} \geq C_{\beta}\left(\frac{r\left(B_{2}\right)}{r\left(B_{1}\right)}\right)^{\varepsilon}
$$

e (i) está provada.

Se $\omega$ é um peso dobrante, e $r>0$, suponha que o anel $A=B(x, 2 r) \backslash B(x, r) \neq \emptyset$. Então existe $y \in A$ e $\alpha>0$ tal que $B(y, \alpha r) \subset A$. Como $B(y, \alpha r) \cap B(x, 2 r) \neq \emptyset$, por (iii) do Corolário 1.14, existe constante $C>0$ tal que

$$
B(x, r) \subset B(x, 2 r) \subset B(y, C \alpha r)
$$

Como por hipótese $\omega$ é dobrante, existe $c_{1}>1$ tal que $\omega(B(y, C \alpha r)) \leq c_{1} \omega(B(y, \alpha r))$. E assim,

$$
\omega(A) \geq \omega(B(y, \alpha r)) \geq \frac{1}{c_{1}} \omega(B(y, C \alpha r)) \geq \frac{1}{c_{1}} \omega(B(x, r)) .
$$

Logo, como $\omega(A)=\omega(B(x, 2 r))-\omega(B(x, r))$,

$$
\omega(B(x, 2 r)) \geq\left(1+\frac{1}{c_{1}}\right) \omega(B(x, r))
$$

Agora tomando $\beta=1+\frac{1}{c_{1}}$, obtemos (ii). 


\subsection{A classe dos pesos $A_{\infty}$}

Agora que já definimos a classe dos pesos $A_{p}$ e conhecemos suas propriedades principais, podemos definir a classe dos pesos $A_{\infty}$.

Definição 2.11. Dizemos que um peso $\omega$ pertence a classe $A_{\infty}$ se $\omega \in \cup_{p \geq 1} A_{p}$.

Temos os seguintes resultados para um elemento da classe $A_{\infty}$ :

Teorema 2.12. São equivalentes as seguintes afirmações para um peso $\omega \in A_{\infty}$ :

(i) Existem $0<\alpha, \beta<1$ tais que para todo cubo $Q$ e todo subconjunto mensurável $E \subset Q$ tem-se: se $|E|<\alpha|Q|$ então $\int_{E} \omega \leq \beta \int_{Q} \omega ;$

(ii) Existem $0<\alpha, \beta<1$ tais que para todo cubo $Q$ e todo subconjunto mensurável $E \subset Q$ tem-se: se $\int_{E} \omega \leq \alpha \int_{Q} \omega$ então $|E|<\beta|Q|$;

(iii) Existem $0<\alpha, \beta<1$ tais que para todo cubo $Q$,

$$
\left|\left\{x \in Q: \omega(x)>\beta \omega_{B}\right\}\right| \geq \alpha|Q|
$$

onde $\omega_{B}=\frac{1}{|Q|} \int_{B} \omega$, como mencionamos no início deste capítulo;

(iv) Existem $r>1$ e $C>0$ tais que para todo cubo $Q$,

$$
\left(\frac{1}{|Q|} \int_{Q} \omega^{r}\right)^{\frac{1}{r}} \leq C \frac{1}{|Q|} \omega(Q) ;
$$

(v) $\omega \in A_{p}$ para algum $1 \leq p<\infty$;

(vi) Existem $\varepsilon>0$ e $C>0$ tais que para todo cubo $Q$ e todo conjunto mensurável $E \subset Q$,

$$
\frac{\omega(E)}{\omega(Q)} \leq C\left(\frac{|E|}{|Q|}\right)^{\varepsilon}
$$

(vii) Existem $\varepsilon>0$ e $C>0$ tais que para todo cubo $Q$ e todo conjunto mensurável $E \subset Q$,

$$
\frac{|E|}{|Q|} \leq C\left(\frac{\omega(E)}{\omega(Q)}\right)^{\varepsilon}
$$


Para demonstrar este teorema precisaremos do lema a seguir.

Lema 2.13 (Calderón-Zygmund). Seja $\omega$ um peso, $Q$ um cubo no $\mathbb{R}^{N}$ e $\lambda>$ $\frac{1}{|Q|} \int_{Q} \omega$. Então existe uma seqüência de cubos que não se sobrepõem $\left\{Q_{i}\right\}$ contidos em $Q$ tais que

(i) $\omega(x) \leq \lambda$, se $x \in Q \backslash \cup_{i} Q_{i}$;

(ii) $\lambda \leq \frac{1}{\left|Q_{i}\right|} \int_{Q_{i}} \omega \leq 2^{N} \lambda$.

Prova: Seja $Q_{0}=Q$. Subdivida $Q_{0}$ em $2^{N}$ subcubos que não se sobrepõem. Denote estes cubos por $\left\{Q^{1}\right\}$. Desta coleção, selecione aqueles cubos $Q^{1}$ tais que

$$
\frac{1}{\left|Q^{1}\right|} \int_{Q^{1}} \omega>\lambda
$$

Se $Q^{1}$ for um dos cubos selecionados, temos

$$
\lambda<\frac{1}{\left|Q^{1}\right|} \int_{Q^{1}} \omega \leq \frac{1}{\left|Q^{1}\right|} \int_{Q_{0}} \omega=\frac{1}{2^{N}\left|Q_{0}\right|} \int_{Q_{0}} \omega<2^{N} \lambda .
$$

Se $Q^{1}$ não for um dos cubos selecionados, então subdivida-o em $2^{N}$ subcubos, $\left\{Q^{2}\right\}$, digamos. Selecione aqueles $Q^{2}$ tais que

$$
\frac{1}{\left|Q^{2}\right|} \int_{Q^{2}} \omega>\lambda
$$

Se $Q^{2}$ for um dos cubos selecionados, temos

$$
\lambda<\frac{1}{\left|Q^{2}\right|} \int_{Q^{2}} \omega \leq \frac{\left|Q^{1}\right|}{\left|Q^{2}\right|} \frac{1}{\left|Q^{1}\right|} \int_{Q_{1}} \omega<2^{N} \lambda .
$$

O processo continua escolhendo-se sempre os subcubos $\left\{Q^{i}\right\}$, com

$$
\frac{1}{\left|Q^{i}\right|} \int_{Q^{i}} \omega>\lambda
$$

A coleção de todos os cubos selecionados é enumerável e quaisquer dois cubos escolhidos não se sobrepõem e (ii) está assegurada pela construção. Agora se $x \in Q$ e não está em nenhum dos cubos selecionados, então, a cada passo, $x$ pertence a um cubo que não foi selecionado. Assim, existe uma seqüência de cubos $Q_{j} \searrow x$ tal que

$$
\frac{1}{\left|Q_{j}\right|} \int_{Q_{j}} \omega \leq \lambda
$$

Portanto, $\omega(x) \leq \lambda$ quase sempre em $Q \backslash \cup_{i} Q^{i}$. 
Prova do Teorema 2.12: Mostremos que (iii) implica (iv). Suponhamos que existam $0<\alpha, \beta<1$ tais que para todo cubo $Q$,

$$
\left|\left\{x \in Q: \omega(x)>\beta \omega_{Q}\right\}\right| \geq \alpha|Q|
$$

Afirmamos que existe $\gamma>0$ tal que para todo $\lambda>\omega_{B}$,

$$
\int_{\{x \in Q: \omega(x)>\lambda\}} \omega \leq C \lambda|\{x \in Q: \omega(x)>\gamma \lambda\}| .
$$

De fato, fixemos $Q$ e $\lambda$ com acima. Pelo Lema 2.13 existe uma seqüência de cubos não sobrepostos $\left\{Q_{i}\right\}$ contidos em $Q$ satisfazendo

(a) $\omega(x) \leq \lambda$ se $x \in Q \backslash \cup_{i} Q^{i}$

(b) $\lambda \leq \frac{1}{\left|Q_{i}\right|} \int_{Q_{i}} \omega \leq 2^{N} \lambda$.

Portanto, $\{x \in Q: \omega(x)>\lambda\} \subset \cup_{i} Q_{i}$, e assim,

$$
\begin{aligned}
\omega(\{x \in Q: \omega(x)>\lambda\}) & \leq \sum_{i} \int_{Q_{i}} \omega=\sum_{i}\left(\frac{1}{\left|Q_{i}\right|} \int_{Q_{i}} \omega\right)\left|Q_{i}\right| \\
& \leq 2^{N} \lambda \sum_{i}\left|Q_{i}\right| \text { por }(\mathrm{b}) \\
& \leq \frac{1}{\alpha} 2^{N} \lambda \sum_{i}\left|\left\{x \in Q_{i}: \omega(x)>\beta \omega_{Q_{i}}\right\}\right|
\end{aligned}
$$

por (iii)

$\leq \frac{1}{\alpha} 2^{N} \lambda \sum_{i}\left|\left\{x \in Q_{i}: \omega(x)>\beta \lambda\right\}\right|$

pelo Lema 2.13

$\leq \frac{1}{\alpha} 2^{N} \lambda|\{x \in Q: \omega(x)>\beta \lambda\}|$

pois os cubos não se sobrepõem. Isto demonstra a afirmação que fizemos $\operatorname{com} C=$ $\frac{1}{\alpha} 2^{N}$ e $\gamma=\beta$.

Assumamos momentaneamente que $\int_{Q} \omega^{r}<\infty$, se $r>1$, suficientemente próximo 
de 1. Temos,

$$
\begin{aligned}
\int_{Q} \omega^{r} & =\int_{Q} \omega^{r-1} \omega \\
& =(r-1) \int_{0}^{\infty} \lambda^{r-2} \omega(\{x \in Q: \omega(x)>\lambda\}) d \lambda \text { pelo Lema } 1.26 \\
& =(r-1) \int_{0}^{\omega_{Q}} \lambda^{r-2} \omega(\{x \in Q: \omega(x)>\lambda\}) d \lambda \\
& +\int_{\omega_{Q}}^{\infty} \lambda^{r-2} \omega(\{x \in Q: \omega(x)>\lambda\}) d \lambda \\
& =I+I I, \text { digamos. }
\end{aligned}
$$

Note que

$$
\begin{aligned}
I & \leq(r-1) \omega(Q) \int_{0}^{\omega_{Q}} \lambda^{r-2} d \lambda \\
& =(r-1) \omega(Q) \frac{1}{(r-1)}\left(\frac{1}{|Q|} \int_{Q} \omega\right)^{r-1} \\
& =|Q|\left(\frac{1}{|Q|} \int_{Q} \omega\right)^{r}
\end{aligned}
$$

e pela afirmação que mostramos,

$$
\begin{aligned}
I I & \leq C(r-1) \int_{\omega_{Q}}^{\infty} \lambda^{r-2} \lambda|\{x \in Q: \omega(x)>\gamma \lambda\}| d \lambda \\
& \leq C(r-1) \int_{0}^{\infty} \lambda^{r-1}|\{x \in Q: \omega(x)>\gamma \lambda\}| d \lambda \\
& \leq C \frac{1}{\gamma^{r}}(r-1) \int_{0}^{\infty} \lambda^{r-1}|\{x \in Q: \omega(x)>\lambda\}| d \lambda \\
& =C \frac{1}{\gamma^{r}} \frac{(r-1)}{r} \int_{Q} \omega^{r}(x) d x .
\end{aligned}
$$

Assim,

$$
\begin{aligned}
\int_{Q} \omega^{r}(x) d x & \leq|Q|\left(\frac{1}{|Q|} \int_{Q} \omega\right)^{r}+C \frac{1}{\gamma^{r}} \frac{(r-1)}{r} \int_{Q} \omega^{r} \\
& \leq|Q|\left(\frac{1}{|Q|} \int_{Q} \omega\right)^{r}+\frac{1}{2} \int_{Q} \omega^{r}
\end{aligned}
$$

se tomarmos $r>1$ suficientemente próximo de 1. Portanto,

$$
\int_{Q} \omega^{r}(x) d x \leq 2|Q|\left(\frac{1}{|Q|} \int_{Q} \omega\right)^{r},
$$

para $r>1$, suficientemente próximo de 1, e daí obtemos

$$
\left(\frac{1}{|Q|} \int_{Q} \omega^{r}\right)^{\frac{1}{r}} \leq C\left(\frac{1}{|Q|} \int_{Q} \omega\right)
$$


que é a condição (iv) no caso em que $\int_{Q} \omega^{r}<\infty$ e $r>1$, suficientemente próximo de 1 .

No caso geral, considere a seqüência de pesos $\omega_{k}=\min \{\omega, k\}, k=1,2, \ldots$ Como $\omega_{k} \leq k$, então

$$
\int_{Q}\left(\omega_{k}\right)^{r}<\infty
$$

para todo $r>0$. Assim, é suficiente aplicar o argumento anterior para $\omega_{k}$ e fazer uso do Teorema da Convergência Monótona no final, contanto que mostremos que $\omega_{k}$ satisfaz (iii) com constantes independentes de $k$. Mas, como $\omega$ satisfaz (iii), é suficiente mostrar que para cada $k$,

$$
\left\{x \in Q: \omega_{k}(x)>\beta\left(\omega_{k}\right)_{Q}\right\} \supset\left\{x \in Q: \omega(x)>\beta \omega_{Q}\right\} .
$$

Seja $x \in\left\{x \in Q: \omega(x)>\beta \omega_{Q}\right\}$. Se $\omega_{k}(x)=\omega(x)$ então $\omega_{k}(x)>\beta \omega_{Q}$, já que $\omega_{k} \leq \omega$. Se $\omega_{k}(x)=k$, então

$$
\omega_{k}(x) \geq \frac{1}{|Q|} \int_{Q} \omega_{k}>\beta\left(\omega_{k}\right)_{Q},
$$

já que $\omega_{k} \leq k$ e $0<\beta<1$. Portanto, $x \in\left\{x \in Q: \omega_{k}(x)>\beta\left(\omega_{k}\right)_{Q}\right\}$, e isso conclui a prova de que (iii) implica (iv)

Mostremos agora que a condição (iv) implica a condição (vi). Assumamos, então, que existem $r>1$ e $C>0$ tais que

$$
\left(\frac{1}{|B|} \int_{Q} \omega^{r}\right)^{\frac{1}{r}} \leq C \frac{1}{|Q|} \int_{Q} \omega
$$

para todo cubo $Q$.

Se $Q$ é um cubo dado, considere $E \subset Q$ um conjunto mensurável. Pela desigualdade de Hölder temos,

$$
\begin{aligned}
\int_{E} \omega=\int_{Q} \chi_{E} \omega & \leq\left(\int_{Q} \chi_{E}\right)^{\frac{1}{r^{\prime}}}\left(\int_{Q} \omega^{r}\right)^{\frac{1}{r}} \\
& =|E|^{\frac{1}{r^{\prime}}}|Q|^{\frac{1}{r}}\left(\frac{1}{|Q|} \int_{Q} \omega^{r}\right)^{\frac{1}{r}} \\
& \leq C|E|^{\frac{1}{r^{\prime}}}|Q|^{\frac{1}{r}} \frac{1}{|Q|} \int_{Q} \omega \text { por (iv) } \\
& =C\left(\frac{|E|}{|Q|}\right)^{\frac{1}{r^{\prime}}} \int_{Q} \omega \\
& =C\left(\frac{|E|}{|Q|}\right)^{\frac{1}{r^{\prime}}} \omega(Q) .
\end{aligned}
$$


Portanto,

$$
\frac{\omega(E)}{\omega(Q)} \leq C\left(\frac{|E|}{|Q|}\right)^{\frac{1}{r^{\prime}}}
$$

que é (vi) $\operatorname{com} \varepsilon=\frac{1}{r^{\prime}}$.

Note que se a condição (vi) for satisfeita para um dado peso $\omega$, então, para todo subconjunto mensurável $E$ de um dado cubo $Q$, tal que $|E|<\alpha|Q|$, onde $0<\alpha<1$ é tal que $\beta=C \alpha^{\varepsilon}<1$ e as constantes $C$ e $\varepsilon$ são as mesmas da condição (vi), temos

$$
\int_{E} \omega \leq C\left(\frac{|E|}{|Q|}\right)^{\varepsilon} \int_{Q} \omega \leq C \alpha^{\varepsilon} \int_{Q} \omega=\beta \int_{Q} \omega
$$

que e é a condição (i).

Agora, se (i) for verdadeira, ou seja, se existirem $0<\alpha, \beta<1$ tais que se $\omega(E)>$ $\beta \omega(Q)$ implicar que $|E| \geq \alpha|Q|$, para todo $E \subset Q$, então para $\widetilde{E}=Q \backslash E \subset Q$, temos

$$
[\omega(\widetilde{E})>\beta \omega(Q) \Rightarrow|\widetilde{E}| \geq \alpha|Q|],
$$

que é equivalente a

$$
[\omega(Q)-\omega(E)>\beta \omega(Q) \Rightarrow|Q|-|E| \geq \alpha|Q|],
$$

ou ainda,

$$
[(1-\beta) \omega(Q)>\omega(E) \Rightarrow(1-\alpha)|Q| \geq|E|] .
$$

E assim, se $\beta^{\prime}=1-\beta$ e $\alpha^{\prime}=1-\alpha$,

$$
\left[\beta^{\prime} \omega(Q)>\omega(E) \Rightarrow \alpha^{\prime}|Q| \geq|E|\right] \text {. }
$$

Portanto, temos que (i) é equivalente à (ii), se considerarmos que $\beta^{\prime}=\alpha$ e $\alpha^{\prime}=\beta$ de (ii).

Suponhamos que (ii) seja válida. Tome $\beta^{\prime} \in(0, \alpha)$, onde $\alpha$ é como em (ii). Seja $Q$ um cubo e $E=\left\{x \in Q: \omega(x)>\beta^{\prime} \omega_{Q}\right\}$. Temos

$$
\begin{aligned}
\omega(Q \backslash E)=\int_{Q \backslash E} \omega & \leq \beta^{\prime} \int_{Q \backslash E} \omega_{Q} \\
& =\beta^{\prime}\left(\frac{1}{|Q|} \int_{Q} \omega\right)\left(\int_{Q \backslash E} 1\right) \\
& \leq \beta^{\prime} \omega(Q) \\
& <\alpha \omega(Q) .
\end{aligned}
$$


Assim, por (ii), $|Q \backslash E|<\beta|Q|$. Portanto,

$$
|E|=|Q|-|Q \backslash E|>|Q|-\beta|Q|=(1-\beta)|Q|=\alpha^{\prime}|Q|,
$$

onde $\alpha^{\prime}=1-\beta$, que é exatamente a condição (iii).

Observe qiıe até agora provamos que são equivalentes as seguintes condições para um peso $\omega$ : (i), (ii), (iii), (iv) e (vi).

Afirmamos que se $\omega$ satisfaz (i) então a medida $d \mu=\omega d x$ é dobrante. De fato, suponhamos que $\omega$ satisfaz (i) e sejam $\alpha$ e $\beta$ como nessa condição. Escolha $\delta>0$ tal que

$$
|(1+\delta) Q \backslash Q|<\alpha|(1+\delta) Q|
$$

Então, por (i), obtemos

$$
\mu((1+\delta) Q \backslash Q)<\beta \mu((1+\delta)) Q)
$$

Assim, $\mu(Q)>(1-\beta) \mu((1+\delta) Q)$ e, portanto,

$$
\mu((1+\delta) Q)<\frac{1}{1-\beta} \mu(Q) .
$$

E iterando, obtemos

$$
\mu(2 Q)<\left(\frac{1}{1-\beta}\right)^{N} \mu(Q),
$$

para algum $N>0$, depende somente de $\delta$. Isto termina a prova da nossa afirmação.

Observe que a condição (ii), em termos da medida $\mu$ introduzida acima, se exprime por: Existem constantes $0<\alpha, \beta<1$ tais que para todo cubo $Q$ e todo subconjunto mensurável $E$ de $Q$ satisfazendo $\mu(E) \leq \alpha \mu(Q)$ tem-se

$$
\int_{E} \omega^{-1} d \mu \leq \beta \int_{Q} \omega^{-1} d \mu
$$

Esta condição é a mesma que (i) com a medida de Lebesgue e $\omega$ trocados por $d \mu$ e $\omega^{-1}$, respectivamente. Denotemos por (n') a condição análoga à condição (n) com a medida de Lebesgue e $\omega$ substituídos por $d \mu$ e $\omega^{-1}$, respectivamente, para $\mathrm{n}=$ i,ii,iii,iv e vi.

Desta forma, se $\omega$ satisfaz a condição (i), por exemplo, então, como já vimos, $d \mu$ é dobrante e como o Lema de Calderón-Zygmund continua verdadeiro para um tal peso, vemos que as condições (n) são equivalentes às condições (n'), para n= i,ii,iii,iv e vi. 
Passemos a demonstrar que (i) implica (v). Assuma que (i) seja satisfeita por $\omega$. Então, por (iv'), existem constantes $C>0$ e $r>1$ tais que para todo cubo $Q$,

$$
\left(\frac{1}{\mu(Q)} \int_{Q} \omega^{-r} d \mu\right)^{\frac{1}{r}} \leq \frac{1}{\mu(Q)} \int_{Q} \frac{1}{\omega} d \mu
$$

Isto é,

$$
\mu(Q)^{1-\frac{1}{r}}\left(\int_{Q} \omega^{1-r}\right)^{\frac{1}{r}} \leq C|Q|
$$

e assim,

$$
\left(\frac{1}{|Q|} \int_{Q} \omega\right)^{\frac{1}{r^{\prime}}}\left(\frac{1}{|Q|} \int_{Q} \omega^{1-r}\right)^{\frac{1}{r}} \leq C,
$$

onde $r^{\prime}$ é o expoente conjugado a $r$, e portanto,

$$
\left(\frac{1}{|Q|} \int_{Q} \omega\right)^{\frac{1}{r^{\prime}}}\left(\frac{1}{|Q|} \int_{Q} \omega^{-\frac{1}{r^{\prime}-1}}\right)^{\frac{1}{r}} \leq C
$$

ou seja, $\omega \in A_{r^{\prime}}$ e a condição (v) está satisfeita por $\omega$.

Reciprocamente, suponhamos que $\omega$ satisfaz (v), isto é, que $\omega \in A_{r}$ para algum $r \geq 1$. Podemos assumir $r>1$. Assim, existe $C>0$ tal que para todo cubo $Q$,

$$
\begin{aligned}
& \left(\frac{1}{|Q|} \int_{Q} \omega\right)\left(\frac{1}{|Q|} \int_{Q} \omega^{-\frac{1}{r-1}}\right)^{r-1} \leq C \\
& \left(\frac{1}{|Q|} \int_{Q} \omega\right)\left(\frac{1}{|Q|} \int_{Q} \omega^{1-r^{\prime}}\right)^{r-1} \leq C \\
& \left(\frac{1}{|Q|} \int_{Q} \omega\right)^{\frac{1}{r}}\left(\frac{1}{|Q|} \int_{Q} \omega^{1-r^{\prime}}\right)^{\frac{1}{r^{\prime}}} \leq C^{\frac{1}{r}} \\
& \left(\int_{Q} \omega\right)^{\frac{1}{r}}\left(\int_{Q} \omega^{-r^{\prime}} \omega\right)^{\frac{1}{r^{\prime}}} \leq C^{\frac{1}{r}}|Q| .
\end{aligned}
$$

Logo,

$$
\mu(Q)^{-\frac{1}{r^{\prime}}}\left(\int_{Q} \omega^{-r^{\prime}} d \mu\right)^{\frac{1}{r^{\prime}}} \leq C^{\frac{1}{r}} \mu(Q)^{-1}|Q|=C^{\frac{1}{r}} \mu(Q)^{-1} \int_{Q} \omega^{-1} d \mu
$$

onde $d \mu=\omega d x$. E assim,

$$
\left(\frac{1}{\mu(Q)} \int_{Q} \omega^{-r^{\prime}} d \mu\right)^{\frac{1}{r^{\prime}}} \leq C^{\frac{1}{r}} \mu(Q)^{-1} \int_{Q} \omega^{-1} d \mu
$$

que é a condição (iv'). 
Já sabemos que (iv') é equivalente a (vi'). Para terminar a demonstração do Teorema 2.12, mostremos que (vi') é equivalente a (vii). Para isso, assumamos que existem $C>0$ e $\varepsilon>0$ tais que para todo cubo $Q$ e todo subconjunto mensurável $E \subset Q$

$$
\frac{\int_{E} \omega^{-1} d \mu}{\int_{Q} \omega^{-1} d \mu} \leq\left(\frac{\mu(E)}{\mu(Q)}\right)^{\varepsilon}
$$

ou seja,

$$
\frac{|E|}{|Q|} \leq C\left(\frac{\omega(E)}{\omega(Q)}\right)^{\varepsilon}
$$

que é a condição (vii).

Observação 2.15. A condição (iv) é conhecida como Hölder reversa.

\subsection{A classe dos pesos $A_{\infty}$-forte}

Agora estamos preparados para estudar a classe dos pesos $A_{\infty}$-forte.

Seja $\omega(z)$ um peso $A_{\infty}$ com relação à medida de Lebesgue. Dados $z_{1}, z_{2} \in \mathbb{R}^{N}$, defina

$$
\varrho\left(z_{1}, z_{2}\right)=\inf _{B: z_{1}, z_{2} \in B}\left(\int_{B} \omega(z) \lambda(z)^{\frac{m}{N-1}} d z\right)^{\frac{1}{N}}
$$

onde $B$ denota uma $\rho$-bola, $\rho$ definida como em $(1.10)$ e $\lambda(z)=\lambda(x), z=(x, y) \in$ $\mathbb{R}^{n+m}, n+m=N, \lambda$ contínua com $\lambda \geq 0$, e $\lambda>0$ exceto possivelmente em um número finito de pontos, satisfazendo a condição $R H_{\infty}$ definida por

$$
\frac{1}{|B|} \int_{B} \lambda(z) d z \approx \operatorname{supess}_{B} \lambda(z)
$$

com constantes de equivalência independentes de $B$.

Se $\gamma:[0, T] \rightarrow \mathbb{R}^{N}$ é uma curva sub-unitária, definimos o $\omega$-comprimento de $\gamma$ por

$$
l(\gamma)=\lim _{|\sigma| \rightarrow 0} \inf \sum_{i=0}^{l-1} \varrho\left(\gamma\left(t_{i+1}\right), \gamma\left(t_{i}\right)\right)
$$

onde $\sigma=\left\{t_{0}, \ldots, t_{l}\right\}$ é uma partição de $[0, T]$ e $|\sigma|$ denota a norma da partição. 
Defina a distância $d\left(z_{1}, z_{2}\right)$ por

$$
d\left(z_{1}, z_{2}\right)^{-}=\inf \left\{l(\gamma): \gamma \text { é uma curva sub-unitária unindo } z_{1} \text { a } z_{2}\right\} \text {. }
$$

Note que na definição de $d$, podemos nos restringir a uma curva sem auto intersecção. Então $d$ é uma pseudo-métrica, isto é, satisfaz as propriedades de métrica, exceto que, $d\left(z_{1}, z_{2}\right)=0$ sem implicar que $z_{1}=z_{2}$.

E agora podemos definir a classe $A_{\infty}$-forte.

Definição 2.19. Seja @ definido como em (2.16), e d como em (2.18). Se existirem constantes positivas $c_{1}, c_{2}$ independentes de $z_{1}$ e $z_{2}$ tais que

$$
c_{1} \varrho\left(z_{1}, z_{2}\right) \leq d\left(z_{1}, z_{2}\right) \leq c_{2} \varrho\left(z_{1}, z_{2}\right)
$$

então d é uma métrica e dizemos que $\omega$ é um peso $A_{\infty}$-forte com relação à métrica d.

E temos o seguinte resultado para elementos satisfazendo a condição (2.17):

Proposição 2.20. Seja $(X, \rho, \mu)$ um espaço homogêneo e seja $\omega \in L_{l o c}^{1}$ e $\omega>0$ quase sempre. Então valem as seguintes propriedades:

(i) $S e \omega \in R H_{\infty}$ então $\omega \in A_{\infty}$;

(ii) $\omega \in R H_{\infty}$ se, e somente se $\omega^{\beta} \in R H_{\infty}$ para todo $\beta>0$;

(iii) $S e \omega \in R H_{\infty}$ e $u \in A_{\infty}$ então $\omega u \in A_{\infty}$.

Prova: Para provar (i), por (v) do Teorema 2.12 basta mostrar que se $\omega \in R H_{\infty}$, então $\omega$ satisfaz a desigualdade de Hölder reversa. Como

$$
\frac{1}{\mu(B)} \int_{B} \omega d \mu \approx \operatorname{supess}_{B} \omega
$$

para todo $r>1$, temos

$$
\begin{aligned}
\left(\frac{1}{\mu(B)} \int_{B} \omega^{r} d \mu\right)^{\frac{1}{r}} & \leq\left(\frac{1}{\mu(B)} \int_{B}\left(\operatorname{supess}_{B} \omega\right)^{r} d \mu\right)^{\frac{1}{r}} \\
& =\operatorname{supess}_{B} \omega\left(\frac{1}{\mu(B)} \int_{B} d \mu\right)^{\frac{1}{r}} \\
& =\operatorname{supess}_{B} \omega \\
& \leq C \frac{1}{\mu(B)} \int_{B} \omega d \mu
\end{aligned}
$$


para toda bola $B$. Portanto $\omega \in A_{\infty}$.

Para provar (ii), note que se $\beta \geq 1$ e $\omega \in R H_{\infty}$, então pela desigualdade de Hölder,

$$
\operatorname{supess}_{B} \omega \leq C \frac{1}{\mu(B)} \int_{B} \omega d \mu \leq c\left(\frac{1}{\mu(B)} \int_{B} \omega^{\beta} d \mu\right)^{\frac{1}{\beta}},
$$

e portanto $\omega^{\beta} \in R H_{\infty}$ e (ii) está provado no caso em que $\beta \geq 1$.

Agora se $\beta<1$ e $\omega \in R H_{\infty}$, pela propriedade (i) que acabamos de provar, temos que $\omega \in A_{\infty}$. Afirmamos que vale a seguinte relação:

$$
\left(\frac{1}{\mu(B)} \int_{B} \omega^{\beta} d \mu\right)^{\frac{1}{\beta}} \approx \frac{1}{\mu(B)} \int_{B} \omega d \mu
$$

De fato, observe que por (iii) do Teorema 2.12, existem constantes positivas $c_{1}, c_{2}$ tais que

$$
\mu\left(\left\{x \in B: \omega(x)>c_{1} \frac{1}{\mu(B)} \int_{B} \omega d \mu\right\}\right) \geq c_{2} \mu(B),
$$

e portanto, como $\frac{1}{\beta}>1$, aplicando a desigualdade de Hölder ao par $\left(\frac{1}{\beta}, \frac{1}{1-\beta}\right)$, obtemos

$$
\begin{aligned}
\frac{1}{\mu(B)} \int_{B} \omega^{\beta} d \mu & \leq \frac{1}{\mu(B)}\left(\int_{B}\left(\omega^{\beta}\right)^{\frac{1}{\beta}} d \mu\right)^{\beta} \mu(B)^{1-\beta} \\
& =\frac{1}{\mu(B)}\left(\int_{B} \omega d \mu\right)^{\beta} \mu(B)^{1-\beta} \\
& =\left(\frac{1}{\mu(B)} \int_{B} \omega d \mu\right)^{\beta} .
\end{aligned}
$$

E assim,

$$
\left(\frac{1}{\mu(B)} \int_{B} \omega^{\beta} d \mu\right)^{\frac{1}{\beta}} \leq \frac{1}{\mu(B)} \int_{B} \omega d \mu .
$$

Para verificar a desigualdade contrária, note que se

$$
A=\left\{x \in B: \omega(x)>\frac{1}{\mu(B)} \int_{B} \omega d \mu\right\}
$$

então,

$$
\begin{aligned}
\int_{B} \omega(x)^{\beta} d \mu(x) & \geq \int_{A} \omega(x)^{\beta} d \mu(x) \\
& >c_{1} \int_{A}\left(\frac{1}{\mu(B)} \int_{B} \omega(z) d \mu(z)\right)^{\beta} d \mu(x) \\
& =c_{1} \mu(A)\left(\frac{1}{\mu(B)} \int_{B} \omega(x) d \mu(x)\right)^{\beta} \\
& \geq C \mu(B)\left(\frac{1}{\mu(B)} \int_{B} \omega(x) d \mu(x)\right)^{\beta} \quad \text { por }(2.22) .
\end{aligned}
$$


Ou seja,

$$
\frac{1}{\mu(B)} \int_{B} \omega d \mu \leq\left(\frac{1}{\mu(B)} \int_{B} \omega^{\beta} d \mu\right)^{\frac{1}{\beta}} .
$$

Assim, (2.21) está provada e, portanto.

$$
\left(\int_{B} \omega^{\beta} d \mu\right)^{\frac{1}{\beta}} \approx \operatorname{supess}_{B} \omega
$$

isto é, $\omega^{\beta} \in R H_{\infty}$.

Para provar (iii), novamente basta mostrar que $\omega u$ satisfaz a desigualdade Hölder reversa. Para isso, escolha $r>1$ e $p>1$ tais que $u \in A_{p}$ e

$$
\frac{1}{\mu(B)} \int_{B} u d \mu \approx\left(\frac{1}{\mu(B)} \int_{B} u^{r} d \mu\right)^{\frac{1}{r}}
$$

Então,

$$
\begin{aligned}
\left(\frac{1}{\mu(B)} \int_{B}(\omega u)^{r} d \mu\right)^{\frac{1}{r}} & \leq\left(\operatorname{supess}_{B} \omega\right)\left(\frac{1}{\mu(B)} \int_{B} u^{r} d \mu\right)^{\frac{1}{r}} \\
& \leq\left(\frac{1}{\mu(B)} \int_{B} \omega^{\frac{1}{p}} d \mu\right)^{p}\left(\frac{1}{\mu(B)} \int_{B} u^{r} d \mu\right)^{\frac{1}{r}} \text { por }(i i) \\
& =\left(\frac{1}{\mu(B)} \int_{B} \omega^{\frac{1}{p}} u^{\frac{1}{p}} u^{-\frac{1}{p}} d \mu\right)^{p} \frac{1}{\mu(B)} \int_{B} u d \mu \text { por }(2.23) \\
& \leq \frac{1}{\mu(B)} \int_{B} \omega u d \mu\left(\frac{1}{\mu(B)} \int_{B} u^{-\frac{1}{p-1}} d \mu\right)^{p-1} \frac{1}{\mu(B)} \int_{B} u d \mu \\
& \leq C \frac{1}{\mu(B)} \int_{B} \omega u d \mu, \text { pois } u \in A_{p} .
\end{aligned}
$$

Portanto $\omega u \in A_{\infty}$ e isso conclui a demonstração.

Observação 2.24. Uma conseqüência imediata da Proposição 2.20 é o fato de que $\mathbb{R}^{N}$ é um espaço homogêneo com relação à $\rho$ e à medida de Lebesgue. De fato, basta usar o seguinte resultado de [FGW]:

Proposição 2.25. Seja $z_{0}=\left(x_{0}, y_{0}\right), t>0, e \Gamma\left(z_{0}, t\right)=\sup _{x:\left|x-x_{0}\right|<t} \lambda(x)\left(\approx \int_{\left|x-x_{0}\right|<t} \lambda(x) d x\right.$ por (2.17), $\Upsilon\left(z_{0}, t\right)=t \Gamma\left(z_{0}, t\right)$. Então as funções $t \rightarrow \Gamma\left(z_{0}, t\right)$ e $t \rightarrow \Upsilon\left(z_{0}, t\right)$ são uniformemente dobrantes com relação a $z_{0}$, isto é, $\Gamma\left(z_{0}, 2 t\right) \leq C \Gamma\left(z_{0}, t\right) e$ $\Upsilon\left(z_{0}, 2 t\right) \leq C \Upsilon\left(z_{0}, t\right)$, com $C$ independente de $z_{0}$ et. 
Considere agora a proposição a seguir, que é resultado do trabalho de [FGW] (ver página 529).

Proposição 2.26. Seja $z_{0}=\left(x_{0}, y_{0}\right)$ e $t>0$. Se $\Gamma$, $\Upsilon$ são como acima e $Q\left(z_{0}, t\right)=$ $B\left(x_{0}, t\right) \times B\left(y_{0}, \Upsilon\left(z_{0}, t\right)\right)$, então existe $b>1$ tal que

$$
Q\left(z_{0}, \frac{t}{b}\right) \subset B\left(z_{o}, t\right) \subset Q\left(z_{0}, b t\right), t>0
$$

Em particular,

$$
\left|B\left(z_{0}, t\right)\right| \approx t^{N} \Gamma\left(z_{0}, t\right)^{m}
$$

Observação 2.28. Note que como $\lambda(x) \in R H_{\infty}$ em $\mathbb{R}^{N}$ com relação à medida de Lebesgue e a distância usual em $\mathbb{R}^{N}$ então por (iii) da Proposição 2.26, $\lambda \in R H_{\infty}$ com relação à métrica $\rho$ e à medida de Lebesgue no $\mathbb{R}^{N}$.

Segue por esta observação que $\omega \lambda^{\frac{m}{N-1}}$ é uma medida dobrante com relação às $\rho$-bolas.

O próximo resultado nos dá uma relação envolvendo pesos $\omega, \lambda \in R H_{\infty}$ que será muito útil na construção do nosso segundo exemplo no Capítulo 4.

Proposição 2.29. Sejam $\omega \in R H_{\infty}$ e $0 \leq \lambda \in R H_{\infty}$ contínua e $d \mu=\omega \lambda^{\frac{m}{N-1}} d z$, onde $N=n+m, n, m \geq 1$. Então,

$$
r(B) \mu(B)^{1-\frac{1}{N}} \approx \int_{B} \omega^{1-\frac{1}{N}} d z
$$

Prova: Como $m, n \geq 1$, então $1-\frac{1}{N}>0$, por (i) e (ii) da Proposição 2.20, temos que $\lambda^{1-\frac{1}{N}} \in R H_{\infty}$. Portanto, por (i) da mesma proposição, $\omega^{1-\frac{1}{N}} \in A_{\infty}$. E então existe $C>0$ tal que

$$
\omega(z) \geq C\left(\frac{1}{\mu(B)} \int_{B} \omega^{1-\frac{1}{N}} d z\right)^{\frac{N}{N-1}}
$$

para todo $z$ em um conjunto $E$ de $B$ cuja medida de Lebesgue seja proporcional a $|B|$, por (iii) do Teorema 2.12, com $\omega^{1-\frac{1}{N}}$ no lugar de $\omega$. Como $\frac{m}{N-1}>0$ e $\lambda \in R H_{\infty}$, temos que $\lambda^{\frac{m}{N-1}} \in R H_{\infty}$, e portanto, $\lambda^{\frac{m}{N-1}} \in A_{\infty}$, pela Proposição 2.20 . 
Conseqüentemente,

$$
\begin{aligned}
r(B) \mu(B)^{1-\frac{1}{N}} & =r(B)\left(\int_{B} \omega \lambda^{\frac{m}{N-1}} d z\right)^{1-\frac{1}{N}} \\
& \geq r(B)\left(\int_{E} \omega \lambda^{\frac{m}{N-1}} d z\right)^{1-\frac{1}{N}} \quad \text { pois } E \subset B \\
& \geq \operatorname{Cr}(B)\left(\int_{E}\left(\frac{1}{\mu(B)} \int_{B} \omega^{1-\frac{1}{N}} d \zeta\right)^{\frac{N}{N-1}} \lambda^{\frac{m}{N-1}} d z\right)^{1-\frac{1}{N}} \\
& =C r(B)\left(\int_{E} \lambda^{\frac{m}{N-1}} d z\right)^{1-\frac{1}{N}}\left(\frac{1}{\mu(B)} \int_{B} \omega^{1-\frac{1}{N}} d \zeta\right) \\
& \geq C r(B)\left(\int_{B} \lambda^{\frac{m}{N-1}} d z\right)^{1-\frac{1}{N}}\left(\frac{1}{\mu(B)} \int_{B} \omega^{1-\frac{1}{N}} d \zeta\right)
\end{aligned}
$$

por (i) do Teorema 2.12. Mas,

$$
\begin{aligned}
r(B)\left(\int_{B} \lambda^{\frac{m}{N-1}} d z\right)^{1-\frac{1}{N}} & =r(B)\left(\frac{1}{|B|} \int_{B} \lambda^{\frac{m}{N-1}} d z\right)^{1-\frac{1}{N}}|B|^{1-\frac{1}{N}} \\
& \approx r(B)\left(\int_{B} \lambda d z\right)^{\frac{m}{N}}|B|^{1-\frac{1}{N}} \quad \text { por }(2.21) \\
& \approx r(B)(\Gamma(z, r))^{\frac{m}{N}}\left[r(B)^{N} \Gamma(z, r)^{m}\right]^{1-\frac{1}{N}} \quad \text { por }(2.27) \\
& \approx r(B)^{N} \Gamma(z, r)^{m} \\
& \approx|B| \quad \operatorname{por}(2.27),
\end{aligned}
$$

onde $B=B(z, r) \mathrm{e}$

$$
\Gamma(z, r)=\operatorname{supess}_{x: x \in B} \lambda(x)\left(\approx \frac{1}{\mu(B)} \int_{B} \lambda(x) d \mu(z) \text { por }(2.17)\right) .
$$

Combinando essas estimativas, obtemos

$$
r(B) \mu(B)^{1-\frac{1}{N}} \geq C \int_{B} \omega^{1-\frac{1}{N}} d z
$$


Para verificar a desigualdade contrária, note que

$$
\begin{aligned}
r(B) \mu(B)^{1-\frac{1}{N}} & =r(B)\left(\int_{B} \omega \lambda^{\frac{m}{N-1}} d z\right)^{1-\frac{1}{N}} \\
& \leq r(B)\left(\max _{B} \lambda\right)^{\frac{m}{N}}\left(\int_{B} \omega d z\right)^{1-\frac{1}{N}} \\
& \leq C r(B)\left(\frac{1}{\mu(B)} \int_{B} \lambda d \mu\right)^{\frac{m}{N}}\left(\int_{B} \omega d z\right)^{1-\frac{1}{N}} \text { pois } \lambda \in R H_{\infty} \\
& =C r(B) \Gamma(z, r)^{\frac{m}{N}}\left(\int_{B} \omega d z\right)^{1-\frac{1}{N}} \\
& \leq C|B|\left(\frac{1}{|B|} \int_{B} \omega d z\right)^{1-\frac{1}{N}} \text { pois } r^{N} \Gamma(z, r)^{m} \approx|B| \\
& \leq C|B| \frac{1}{|B|} \int_{B} \omega^{1-\frac{1}{N}} d z \text { por }(2.27), \text { já que } \omega \in A_{\infty} \\
& =C \int_{B} \omega^{1-\frac{1}{N}} d z .
\end{aligned}
$$

Portanto (2.30) está provada. 


\section{Capítulo 3}

\section{Desigualdades de Poincaré e Fórmulas de Representação}

Neste capítulo estudaremos a relação entre a validade das versões das desigualdades de Poincaré e a existência de fórmulas de representação para funções como as transformações integrais de campos vetoriais de primeira ordem.

Um exemplo mais simples de uma fórmula de representação que temos em mente é a desigualdade para uma função suave $f(x)$ com valores reais definida sobre uma bola $B$ no espaço Euclidiano $\mathbb{R}^{N}$ :

$$
\left|f(x)-f_{B}\right| \leq C \int_{B} \frac{|\nabla f(y)|}{|x-y|^{N+1}} d y, \quad x \in B,
$$

onde $\nabla f$ denota o gradiente de $f, f_{B}$ é a média $\frac{1}{|B|} \int_{B} f(y) d y,|B|$ é a medida de Lebesgue de $B$, e $C$ é uma constante independente de $f, x$ e $B$.

Inicialmente estamos interessados em mostrar que vários análogos da fórmula acima para sistemas mais gerais de campos vetoriais de primeira ordem $X f=$ $\left(X_{1} f, \ldots, X_{m} f\right)$ são conseqüências da desigualdade de Poincaré em $L^{1}$ da forma

$$
\frac{1}{\nu(B)} \int_{B}\left|f-f_{B, \nu}\right| d \nu \leq C r(B) \frac{1}{\mu(B)} \int_{B}|X f| d \mu .
$$

Aqui $\nu$ e $\mu$ são medidas, $B$ é uma bola de raio $r(B)$ com relação à métrica associada aos campos vetoriais, e $f_{B, \nu}=\frac{1}{\nu(B)} \int_{B} f d \nu$.

As fórmulas de representação no $\mathbb{R}^{N}$ foram estudadas para campos vetoriais de Hörmander em [FLW2] e também, para alguns campos vetoriais suaves do tipo Grushin em [FGW], como veremos a seguir. No caso dos campos vetoriais de 
Hörmander, se $\rho(x, y)$ denota a métrica associada e $B=B\left(x_{B}, r\right)$ denota a bola com relação a esta métrica com centro $x_{B}$ e raio $r$, a fórmula de representação fica

$$
\left|f(x)-f_{B}\right| \leq C \int_{\tau B}|X f(y)| \frac{\rho(x, y)}{|B(x, \rho(x, y))|} d y,
$$

para $x \in B$ e $\tau>1$, onde $\tau B$ é a bola com centro $x_{B}$ e raio $\operatorname{\tau r}(B)$.

Como veremos, a equivalência entre estimativas como (3.2) e (3.3) são válidas em vários contextos, e podem ser aplicadas a situações diferentes. A técnica que usaremos para mostrar que desigualdades de Poincaré implicam uma fórmula de representação é um argumento de [FLW2] e o método funciona em qualquer espaço homogêneo. Além disso, o método não requer a presença de um operador derivação $X f$ no lado direito de (3.2) ou na fórmula de representação (3.3): qualquer função poderia ser usada.

\subsection{Desigualdade de Poincaré usual}

Iniciaremos provando a desigualdade de Poincaré usual (3.1) para uma função $f \in \operatorname{Lip}(\bar{B}), B \subset \mathbb{R}^{N}$.

Teorema 3.4. Se $f \in \operatorname{Lip}(\bar{B})$, com $B \subset \mathbb{R}^{N}$, então

$$
\left|f(x)-f_{B}\right| \leq c_{N} \int_{B} \frac{|\nabla f(y)|}{|x-y|^{N-1}} d y
$$

para todo $x \in B$.

Prova: Escreva

$$
\begin{aligned}
\left|f(x)-f_{B}\right| & =\left|f(x)-\frac{1}{|B|} \int_{B} f(y) d y\right|=\left|\frac{1}{|B|} \int_{B}(f(x)-f(y)) d y\right| \\
& \leq \frac{1}{|B|} \int_{B}|f(x)-f(y)| d y
\end{aligned}
$$

Como

$$
\begin{aligned}
|f(y)-f(x)| & =\left|\int_{0}^{1} \frac{d}{d t}\{f(t y+(1-t) x)\} d t\right| \\
& =\left|\int_{0}^{1}(\nabla f)(t y+(1-t) x)(y-x) d t\right| \\
& \leq\left(\int_{0}^{1}|(\nabla f)(t y+(1-t) x)| d t\right)|y-x|
\end{aligned}
$$


segue que

$$
\begin{aligned}
\frac{1}{|B|} \int_{B}|f(x)-f(y)| d y & \leq \frac{1}{|B|} \int_{B}\left(\int_{0}^{1}|(\nabla f)(t y+(1-t) x)| d t\right) d y \\
& =\frac{1}{|B|} \int_{0}^{1} \int_{B}|(\nabla f)(t y+(1-t) x)||x-y| d y d t
\end{aligned}
$$

Note que se $z=t y+(1-t) x$ então $|z-x|=t|y-x|$ e se $r$ é o raio da bola $B$, temos que $\frac{|z-x|}{t}=|y-x| \leq 2 r$, e portanto, $t \geq \frac{|z-x|}{2 r}$.

Combinando esses fatos com a última desigualdade obtemos

$$
\begin{aligned}
\frac{1}{|B|} \int_{B}|f(x)-f(y)| d y & \leq \frac{1}{|B|} \int_{0}^{1}\left(\int_{\{z \in B ;|x-z| \leq 2 r t\}}|(\nabla f)(z)| \frac{|z-x|}{t^{N+1}} d z\right) d t \\
& \leq \frac{1}{|B|} \int_{0}^{\infty}\left(\int_{\{z \in B ;|x-z| \leq 2 r t\}}|(\nabla f)(z)| \frac{|z-x|}{t^{N+1}} d z\right) d t \\
& =\frac{1}{|B|} \int_{B}|(\nabla f)(z)||z-x|\left(\int_{\frac{|x-z|}{2 r}}^{\infty} \frac{d t}{t^{n+1}}\right) d z \\
& =\frac{1}{N} \frac{1}{|B|} \int_{B}|(\nabla f)(z)||z-x|\left(\frac{1}{|z-x|^{N}}(2 r)^{N}\right) d z \\
& =c_{N} \int_{B} \frac{|(\nabla f)(z)|}{|x-z|^{N-1}} d z
\end{aligned}
$$

como queríamos.

\subsection{Condições necessárias para a existência de fór- mulas de representação}

Seja $(\mathcal{S}, \rho, m)$ um espaço homogêneo, onde $\rho$ denota uma quasi-métrica com constante quasi-métrica igual a $k$, isto é, para todo $x, y, z \in \mathcal{S}$,

$$
\rho(x, y) \leq k[\rho(x, y)+\rho(z, y)]
$$

e $m$ é uma medida dobrante. Por definição, $B(x, r)=\{y \in \mathcal{S}: \rho(x, y)<r\}$ denota a bola de centro $x$ e raio $r, m(B(x, r))$ denota a $m$-medida de $B(x, r)$ e, se $B$ é uma bola qualquer, escrevemos $x_{B}$ para o seu centro e $C B$ para a bola de raio $\operatorname{Cr}(B)$ com o mesmo centro $x_{B}$.

Agora vamos apresentar um teorema que nos diz quando desigualdades de Poincaré implicam uma fórmula de representação. 
Teorema 3.5. Sejam $\nu, \rho$ medidas dobrantes em um espaço homogêneo $(\mathcal{S}, \rho, \mu)$. Seja $B_{0}$ uma bola na métrica $\rho, \tau>1, \varepsilon>0$, e assuma que para toda bola $B \subset \tau k B_{0}$ ( $k$ é a constate quasi-métrica),

$$
\frac{1}{\nu(B)} \int_{B}\left|f-f_{B, \nu}\right| d \nu \leq C r(B) \frac{1}{\mu(B)} \int_{B}|X f| d \mu,
$$

onde $f$ é uma função contínua em $\tau k B_{0}$, e que para todas as bolas $\widetilde{B}, B$ com $\widetilde{B} \subset B \subset \tau k B_{0}$,

$$
\frac{r(B)}{\mu(B)} \leq C\left(\frac{r(\widetilde{B})}{r(B)}\right)^{\varepsilon} \frac{r(\widetilde{B})}{\mu(\widetilde{B})},
$$

ou equivalentemente,

$$
\mu(B) \geq C\left(\frac{r(B)}{r(\widetilde{B})}\right)^{\varepsilon+1} \mu(\widetilde{B}) .
$$

Então, para $\nu$-quase todo $x \in B_{0}$,

$$
\left|f(x)-f_{B_{0}, \nu}\right| \leq C \int_{\tau k B_{0}}|X f(y)| \frac{\rho(x, y)}{\mu(B(x, \rho(x, y)))} d \mu(y),
$$

$\operatorname{com} f_{B_{0}, \nu}=\frac{1}{\nu\left(B_{0}\right)} \int_{B_{0}} f d \nu$

No lado direito de (3.6) e (3.8), estamos utilizando a notação vetorial $X f$, mas qualquer função poderia ser utilizada; veja a primeira observação que segue a prova deste teorema, para uma forma mais geral do Teorema 3.5.

Prova: Por hipótese, fixando $\tau>1$, temos, para toda bola $B \subset \tau k B_{0}$, que

$$
\frac{1}{\nu(B)} \int_{B}\left|f-f_{B}\right| d \nu \leq C \frac{r(B)}{\mu(B)} \int_{B}|X f| d \mu,
$$

onde por simplicidade escreveremos $f_{B}$ por $f_{B, \nu}$. Seja $x \in B_{0}$. Afirmamos que existe uma constante $\eta>0$, independente de $x$ e $B_{0}$, tal que $B\left(x, \eta r\left(B_{0}\right)\right) \subset \tau k B_{0}$. De fato, escolha $\eta$ tal que $\eta+1<\tau$. Então, se $y \in B\left(x, \eta r\left(B_{0}\right)\right)$, isto é, $\rho(x, y) \leq \eta r\left(B_{0}\right)$, temos

$$
\begin{aligned}
\rho\left(x_{B_{0}}, y\right) & \leq k\left(\rho\left(x_{B_{0}}, x\right)+\rho(x, y)\right) \quad \text { com } x \in B_{0} \\
& \leq k\left(r\left(B_{0}\right)+\eta r\left(B_{0}\right)\right) \\
& \leq k r\left(B_{0}\right)(1+\eta) \\
& <k \tau r\left(B_{0}\right) .
\end{aligned}
$$


E portanto $y \in \tau k B_{0}$. Denote $B\left(x, \eta r\left(B_{0}\right)\right)=B_{1}$. Então $r=r\left(B_{1}\right)=\eta r\left(B_{0}\right)$. Agora,

$$
\left|f(x)-f_{B_{0}}\right| \leq\left|f(x)-f_{B_{1}}\right|+\left|f_{B_{1}}-f_{B_{0}}\right|
$$

Para o segundo termo do lado direito de (3.9), temos

$$
\begin{aligned}
\left|f_{B_{1}}-f_{B_{0}}\right| & \leq\left|f_{B_{1}}-f_{\tau k B_{0}}\right|+\left|f_{B_{0}}-f_{\tau k B_{0}}\right| \\
& =\left|\frac{1}{\nu\left(B_{1}\right)} \int_{B_{1}} f(y) d \nu(y)-f_{\tau k B_{0}}\right|+\left|\frac{1}{\nu\left(B_{0}\right)} \int_{B_{0}} f(y) d \nu(y)-f_{\tau k B_{0}}\right| \\
& \leq \frac{1}{\nu\left(B_{1}\right)} \int_{B_{1}}\left|f(y)-f_{\tau k B_{0}}\right| d \nu(y)+\frac{1}{\nu\left(B_{0}\right)} \int_{B_{0}}\left|f(y)-f_{\tau k B_{0}}\right| d \nu(y) \\
& \leq\left(\frac{1}{\nu\left(B_{1}\right)}+\frac{1}{\nu\left(B_{0}\right)}\right) \int_{\tau k B_{0}}\left|f(y)-f_{\tau k B_{0}}\right| d \nu(y) \\
& \text { pois } B_{1}, B_{0} \subset \tau k B_{0} \\
& \leq \frac{C}{\nu\left(\tau k B_{0}\right)} \int_{\tau k B_{0}}\left|f(y)-f_{\tau k B_{0}}\right| d \nu(y) \\
& \text { pois } r\left(B_{1}\right), r\left(B_{0}\right) \approx r\left(\tau k B_{0}\right) \text { e } \nu \text { é dobrante } \\
& \leq C \frac{r\left(\tau k B_{0}\right)}{\mu\left(\tau k B_{0}\right)} \int_{\tau k B_{0}}|X f(y)| d \mu(y) \quad \text { pela hipótese (3.6). }
\end{aligned}
$$

E portanto,

$$
\left|f_{B_{1}}-f_{B_{0}}\right| \leq C \int_{\tau k B_{0}}|X f(y)| \frac{\rho(x, y)}{\mu(B(x, \rho(x, y)))} d \mu(y)
$$

onde esta última desigualdade será justificada a seguir. Para $y \in \tau k B_{0}, \rho(x, y) \leq$ $2 k r\left(\tau k B_{0}\right) \approx r\left(B_{0}\right)$. De fato,

$$
\begin{aligned}
\rho(x, y) & \leq k\left(\rho\left(x, x_{B_{0}}\right)+\rho\left(x_{B_{0}}, y\right)\right) \\
& \leq k\left(r\left(B_{0}\right)+r\left(\tau k B_{0}\right)\right) \\
& \leq k\left(r\left(\tau k B_{0}\right)+r\left(\tau k B_{0}\right)\right) \\
& =2 k r\left(\tau k B_{0}\right) .
\end{aligned}
$$

Se para $B=\tau k B_{0}$ e $\widetilde{B}=B\left(x, \frac{(\tau-1) \rho(x, y)}{2 k^{2} \tau}\right)$ mostrarmos que $\widetilde{B} \subset B$, então pela hipótese (3.7) com $\tau k B_{0}$ e $B\left(x, \frac{(\tau-1) \rho(x, y)}{2 k^{2} \tau}\right)$ no lugar de $B$ e $\widetilde{B}$, respectivamente, e 
o fato de $\mu$ ser dobrante, obtemos

$$
\begin{aligned}
\frac{r\left(\tau k B_{0}\right)}{\mu\left(\tau k B_{0}\right)} & \leq C\left(\frac{(\tau-1) \rho(x, y)}{2 k^{2} \tau} \frac{1}{r\left(\tau k B_{0}\right)}\right)^{\varepsilon} \frac{(\tau-1) \rho(x, y)}{2 k^{2} \tau} \frac{1}{c_{1} \mu(B(x, \rho(x, y)))} \\
& \leq C\left(\frac{(\tau-1) 2 k r\left(\tau k B_{0}\right)}{2 k^{2} \tau r\left(\tau k B_{0}\right)}\right)^{\varepsilon} \frac{1}{2 k^{2} \tau c_{1}} \frac{\rho(x, y)}{\mu(B(x, \rho(x, y)))} \\
& =C\left(\frac{(\tau-1)}{\tau k}\right)^{\varepsilon} \frac{1}{2 k^{2} \tau c_{1}} \frac{\rho(x, y)}{\mu(B(x, \rho(x, y)))} \\
& =C \frac{\rho(x, y)}{\mu(B(x, \rho(x, y)))},
\end{aligned}
$$

justificando a desigualdade (3.10). Para mostra que $\widetilde{B} \subset \tau k B_{0}$, consideremos $z \in \widetilde{B}$. Então, lembrando que $\rho(x, y) \leq 2 k r\left(\tau k B_{0}\right)$ quando $y \in \tau k B_{0}$, podemos escrever

$$
\begin{aligned}
\rho\left(x_{B_{0}}, z\right) & \left.\leq k\left(\rho\left(x_{B_{0}}, x\right)+\rho(x, z)\right)\right) \\
& \leq k\left(r\left(B_{0}\right)+\frac{(\tau-1) \rho(x, y)}{2 k^{2} \tau}\right) \\
& \leq k\left(r\left(B_{0}\right)+\frac{(\tau-1) 2 k^{2} \tau r\left(B_{0}\right)}{2 k^{2} \tau}\right) \\
& \leq k\left(r\left(B_{0}\right)+\tau r\left(B_{0}\right)-r\left(B_{0}\right)\right) \\
& =\tau k r\left(B_{0}\right) .
\end{aligned}
$$

E isso mostra que $z \in \tau k B_{0}$.

Para o primeiro termo do lado direito de (3.9), podemos assumir que

$$
\lim _{s \rightarrow 0} f_{B(x, s)}=f(x)
$$

pois esta propriedade vale para $\nu$-quase todo $x$, como pode ser verificado à página 
13 de [S]. Assim, temos

$$
\begin{aligned}
\left|f(x)-f_{B_{1}}\right| & =\left|f(x)-f_{B(x, r)}\right| \\
& \leq \sum_{j=0}^{\infty}\left|f_{B\left(x, r 2^{-j-1}\right)}-f_{B\left(x, r 2^{-j}\right)}\right| \\
& =\sum_{j=0}^{\infty}\left|\frac{1}{\nu\left(B\left(x, r 2^{-j-1}\right)\right)} \int_{B\left(x, r 2^{-j-1}\right)} f(y) d \nu(y)-f_{B\left(x, r 2^{-j}\right)}\right| \\
& \leq \sum_{j=0}^{\infty} \frac{C}{\nu\left(B\left(x, r 2^{-j}\right)\right)} \int_{B\left(x, r 2^{-j}\right)}\left|f(y)-f_{B\left(x, r 2^{-j}\right)}\right| d \nu(y) \\
& \text { pois } \nu \text { é dobrante } \\
& \leq \sum_{j=0}^{\infty} \frac{C r 2^{-j}}{\mu\left(B\left(x, r 2^{-j}\right)\right)} \int_{B\left(x, r 2^{-j}\right)}|X f| d \mu(y) \\
& \text { pela hipótese }(3.6) \\
& =C \int_{B}|X f(y)|\left\{\sum_{j=0}^{\infty} \frac{r 2^{-j}}{\mu\left(B\left(x, r 2^{-j}\right)\right)} \chi_{\left\{y: \rho(x, y)<r 2^{-j}\right\}}(y)\right\} d \mu(y) .
\end{aligned}
$$

Se $\rho(x, y)<r 2^{-j}$, então pela hipótese (3.7), com $B=B\left(x, r 2^{-j}\right)$ e $\widetilde{B}=B(x, \rho(x, y))$, (note que $\widetilde{B} \subset B$ ), obtemos

$$
\frac{r 2^{-j}}{\mu\left(B\left(x, r 2^{-j}\right)\right)} \leq C\left(\frac{\rho(x, y)}{r 2^{-j}}\right)^{\varepsilon} \frac{\rho(x, y)}{\mu(B(x, \rho(x, y)))} .
$$

Assim,

$$
\begin{aligned}
& \sum_{j \geq 0: 2^{j} \leq r \rho(x, y)^{-1}}\left(\frac{2^{j} \rho(x, y)}{r}\right)^{\varepsilon} \frac{\rho(x, y)}{\mu(B(x, \rho(x, y)))} \\
& =C\left(\frac{\rho(x, y)}{r}\right)^{\varepsilon} \frac{\rho(x, y)}{\mu(B(x, \rho(x, y)))} \sum_{j \geq 0: 2^{j} \leq r \rho(x, y)^{-1}}\left(2^{\varepsilon}\right)^{j} .
\end{aligned}
$$

Mas $\sum_{j \geq 0: 2^{j} \leq r \rho(x, y)^{-1}}\left(2^{\varepsilon}\right)^{j}$ é uma série geométrica. Como $j \leq \log _{2}\left(r \rho(x, y)^{-1}\right)$, podemos escrever

$$
\sum_{j \geq 0: 2^{j} \leq r \rho(x, y)^{-1}}\left(2^{\varepsilon}\right)^{j} \leq c_{\epsilon}\left(2^{\epsilon}\right)^{\log _{2}\left(r \rho(x, y)^{-1}\right)}=c_{\epsilon}\left(\frac{r}{\rho(x, y)}\right)^{\epsilon} .
$$

E portanto,

$$
\begin{aligned}
\sum_{j \geq 0: 2^{j} \leq r \rho(x, y)^{-1}}\left(\frac{2^{j} \rho(x, y)}{r}\right)^{\varepsilon} & \frac{\rho(x, y)}{\mu(B(x, \rho(x, y)))} \\
\leq & C\left(\frac{\rho(x, y)}{r}\right)^{\varepsilon} \frac{\rho(x, y)}{\mu(B(x, \rho(x, y)))}\left(\frac{r}{\rho(x, y)}\right)^{\varepsilon} \\
& =C \frac{\rho(x, y)}{\mu(B(x, \rho(x, y)))} .
\end{aligned}
$$


Assim,

$$
\left|f(x)-f_{B_{1}}\right| \leq C \int_{\tau k B_{0}}|X f(y)| \frac{\rho(x, y)}{\mu(B(x, \rho(x, y)))} d \mu(y) .
$$

Agora, aplicando (3.10) e (3.11) em (3.9), provamos o teorema.

Observação 3.12. Como mostra a prova do Teorema 3.5, podemos trocar (3.6) por

$$
\frac{1}{\nu(B)} \int_{B}\left|f-f_{B, \nu}\right| d \nu \leq C \phi(B) \sigma(B)
$$

e (3.7) por

$$
\phi(B) \leq\left(\frac{r(\widetilde{B})}{r(B)}\right)^{\varepsilon} \phi(\widetilde{B}), \quad \widetilde{B} \subset c_{1} B,
$$

onde $\phi$ é uma função não negativa qualquer de bolas $B, c_{1}$ é uma constante suficientemente grande dependendo somente de $\tau$ e $k$, e $\sigma$ é uma medida qualquer, obtendo como conclusão, que para $\nu$-quase todo $x \in B_{0}$,

$$
\left|f(x)-f_{B_{0}, \nu}\right| \leq C \int_{\tau k B_{0}} \phi(B(x, \rho(x, y))) d \sigma(y) .
$$

Observação 3.13. A conclusão do Teorema 3.5 pode ser modificada como a seguir: se $\beta \in(0,1)$ é tal que

$$
\nu\left(E_{f}\right):=\nu\left(\left\{y \in B_{0}: f(y)=0\right\}\right) \geq \beta \nu\left(B_{0}\right),
$$

para quase todo $x \in B_{0}$, temos

$$
|f(x)| \leq C_{\beta} \int_{\tau k B_{0}}|X f(y)| \frac{\rho(x, y)}{\mu(B(x, \rho(x, y)))} d \mu(y) .
$$

De fato, por (3.6),

$$
\begin{aligned}
\frac{r\left(B_{0}\right)}{\mu\left(B_{0}\right)} \int_{B_{0}}|X f| d \mu & \geq C \frac{1}{\nu\left(B_{0}\right)} \int_{B_{0}}\left|f-f_{B_{0}, \nu}\right| d \nu \\
& \geq C \frac{1}{\nu\left(B_{0}\right)} \int_{E_{f}}\left|f-f_{B_{0}, \nu}\right| d \nu \text { pois } E_{f} \subset B_{0} \\
& =C \frac{1}{\nu\left(B_{0}\right)} \int_{E_{f}}\left|f_{B_{0}, \nu}\right| d \nu \\
& =C \frac{\nu\left(E_{f}\right)}{\nu\left(B_{0}\right)}\left|f_{B_{0}, \nu}\right| \\
& \geq C \frac{\nu\left(E_{0}\right)}{\nu\left(E_{f}\right)} \beta\left|f_{B_{0}, \nu}\right| \\
& =C_{\beta}\left|f_{B_{0}, \nu}\right|
\end{aligned}
$$


e então por (3.8),

$$
\begin{aligned}
|f(x)| & \leq\left|f(x)-f_{B_{0}}\right|+\left|f_{B_{0}}\right| \\
& \leq C \int_{\tau k B_{0}}|X f(y)| \frac{\rho(x, y)}{\mu(B(x, \rho(x, y)))} d \mu(y)+\frac{1}{C_{\beta}} \frac{r\left(B_{0}\right.}{\mu\left(B_{0}\right)} \int_{B_{0}}|X f(y)| d \mu(y) \\
& \leq C_{\beta} \int_{\tau k B_{0}}|X f(y)| \frac{\rho(x, y)}{\mu(B(x, \rho(x, y)))} d \mu(y),
\end{aligned}
$$

pelo mesmo argumento utilizado na segunda parte de (3.9).

\subsection{Condições necessárias para desigualdades do tipo de Poincaré}

Como mencionamos anteriormente, a implicação contrária do Teorema 3.5, a saber, resultados mostrando quando fórmulas de representação implicam estimativas de Poincaré em $L^{1}$, pode ser obtida facilmente usando o Teorema de Fubini, como mostra o teorema a seguir.

Teorema 3.14. Seja $\phi(B)$ uma função não-negativa de bolas $B$ em um espaço quasi-métrico $(\mathcal{S}, \rho)$, e sejam $\nu, \sigma$ medidas. Dados $\tau \geq 1$ e uma bola $B$ fixa, suponha que exista uma constante $c_{B}$ tal que

$$
\left|f(x)-c_{B}\right| \leq C \int_{\tau B} \phi(B(x, \rho(x, y))) d \sigma(y), \quad \text { para } \nu \text {-quase todo } x \in B
$$

e que $\phi$ satisfaça a relação

$$
\frac{1}{\nu(B)} \int_{B} \phi(B(x, \rho(x, y))) d \nu(x) \leq C \phi(B), \quad \text { para } \sigma \text {-quase todo } y \in \tau B \text {. }
$$

Então,

$$
\frac{1}{\nu(B)} \int_{B}\left|f(x)-c_{B}\right| d \nu(x) \leq C \phi(B) \sigma(\tau B)
$$

Antes de provarmos o teorema, note que se $(\mathcal{S}, \rho)$ é um espaço métrico e $\nu$ é uma medida dobrante, fixando uma bola $B_{0}$ em $\mathcal{S}$ satisfazendo a condição de Boman $\mathcal{B}(\tau, \varsigma)$, se (3.17) for satisfeita para toda bola $B \operatorname{com} \tau B \subset B_{0}$, e se

$$
\frac{\nu(B)}{\nu\left(B_{0}\right)} \leq C \frac{\phi\left(B_{0}\right)}{\phi(B)}, \text { para toda } B \operatorname{com} \tau B \subset B_{0},
$$


podemos concluir que

$$
\frac{1}{\nu\left(B_{0}\right)} \int_{B_{0}}\left|f(x)-f_{B_{0}, \nu}\right| d \nu(x) \leq C \phi\left(B_{0}\right) \sigma\left(B_{0}\right)
$$

E pelos Teoremas (5.2) e (5.4) de [FGW], página 592, temos que

$$
\frac{1}{\left|B_{0}\right|} \int_{B_{0}}\left|f(x)-f_{B_{0}}\right| d x \leq C r\left(B_{0}\right)\left(\frac{1}{\left|B_{0}\right|} \int_{B_{0}}|X f(x)| d x\right) .
$$

De fato,

$$
\begin{aligned}
\int_{B}\left|f(x)-c_{B}\right| d \nu(x) & \leq C \nu(B) \phi(B) \sigma(\tau B) \\
& \leq C \phi\left(B_{0}\right) \nu\left(B_{0}\right) \sigma(\tau B) \\
& =A \sigma(\tau B),
\end{aligned}
$$

com $A=\phi\left(B_{0}\right) \nu\left(B_{0}\right)$, para toda bola $B$ tal que $\tau B \subset B_{0}$.

Agora pelos Teoremas 5.2 e 5.4 de [FGW], existe uma constante $f_{B_{0}, \nu}$ tal que

$$
\int_{B_{0}}\left|f(x)-f_{B_{0}, \nu}\right| d \nu(x) \leq C \phi(B) \nu\left(B_{0}\right) \sigma\left(B_{0}\right)
$$

e (3.19) está provada.

Prova: Integrando (3.15) com relação a $\nu$ em $B$ e trocando a ordem de integração obtemos

$$
\begin{aligned}
\frac{1}{\nu(B)} \int_{B}\left|f(x)-c_{B}\right| d \nu(x) & \leq \frac{C}{\nu(B)} \int_{B}\left(\int_{\tau B} \phi(B(x, \rho(x, y))) d \sigma(y)\right) d \nu(x) \\
& =C \int_{\tau B}\left(\frac{1}{\nu(B)} \int_{B} \phi(B(x, \rho(x, y))) d \nu(x)\right) d \sigma(y) \\
& \leq C \int_{\tau B} \phi(B) d \sigma(y), \quad \text { por }(3.16) \\
& =C \phi(B) \sigma(\tau B),
\end{aligned}
$$

e o teorema está provado. 


\section{Capítulo 4}

\section{Aplicações}

Finalmente construiremos alguns exemplos e aplicações relacionando os Teoremas (3.5) e (3.14)

Estes exemplos foram apresentados em [FLW1], o artigo que serviu de base para este trabalho, e para verificarmos as hipóteses dos Teoremas citados acima, foi necessário estudar alguns resultados de [FGW], [FLW2], [J] e [NSW].

\subsection{Equivalência da desigualdade de Poincaré e existência de fórmulas de representação para campos vetoriais de Hörmander}

Sejam $X_{1}, \ldots, X_{q}$ uma coleção de campos vetoriais satisfazendo a condição de Hörmander. Considere o espaço homogêneo $\left(\mathbb{R}^{N}, \rho, d x\right)$, onde $\rho$ a métrica associada a estes campos vetoriais e $d x$ é a medida de Lebesgue.

Então pelo trabalho de [J] temos (3.6) satisfeita com $|X f|=\sum_{i=1}^{M}\left|X_{i} f\right|$ e $d \mu=$ $d \nu=d x$.

Também (3.7) está satisfeita com $d \mu=d x$ e $\varepsilon=N-1$ pelo Corolário 1.20. De fato, pela primeira desigualdade em (1.21), com $B=B(x, r)$ e $\widetilde{B}=B(x, s), r, s$ como naquele corolário, obtemos

$$
\mu(B) \geq C_{1}\left(\frac{r(B)}{r(\widetilde{B})}\right)^{\varepsilon+1} \mu(\widetilde{B})
$$


Assim, pelo Teorema 3.5, vale a fórmula de representação, ou seja,

$$
\left|f(x)-f_{B_{0}, \nu}\right| \leq C \int_{\tau k B_{0}}|X f(y)| \frac{\rho(x, y)}{\mu(B(x, \rho(x, y)))} d \mu(y) .
$$

Reciprocamente, com relação ao Teorema 3.14, note que se tomarmos

$$
\phi(B)=\frac{r(B)}{|B|}, d \sigma=|X f| d x \text { e } d \nu=d \mu=d x
$$

então fixando $y \in \tau B$, como $\rho(x, y) \leq C r(B)$ quando $x \in B$, segue que

$$
I=\frac{1}{\nu(B)} \int_{B} \phi(B(x, \rho(x, y))) d \nu(x)=\frac{1}{|B|} \int_{B} \frac{\rho(x, y)}{|B(x, \rho(x, y))|} d x
$$

e portanto,

$$
\begin{aligned}
I & \leq \frac{1}{|B|} \sum_{j=0}^{\infty}\left(\int_{C 2^{-j} r(B) \leq \rho(x, y) \leq C 2^{-j+1} r(B)} d x\right) \frac{C 2^{-j+1} r(B)}{\left|B\left(x, C 2^{-j} r(B)\right)\right|} \\
& \leq \frac{1}{|B|} \sum_{j=0}^{\infty} \frac{\left|B\left(x, C 2^{1-j} r(B)\right)\right|}{\left|B\left(x, C 2^{-j} r(B)\right)\right|} C 2^{1-j} r(B) \\
& \leq \frac{2}{|B|} C r(B) \sum_{j=0}^{\infty} \frac{\left|B\left(x, C 2^{-j} r(B)\right)\right|}{\left|B\left(x, C 2^{-j} r(B)\right)\right|}\left(\frac{1}{2}\right)^{j} \\
& =\frac{2}{|B|} C r(B) \sum_{j=0}^{\infty}\left(\frac{1}{2}\right)^{j} \\
& =C \frac{r(B)}{|B|} \\
& =C \phi(B) .
\end{aligned}
$$

Portanto a hipótese (3.16) está verificada.

Agora só nos resta provar que a hipótese (3.15) também é válida, ou seja, queremos obter uma fórmula de representação para uma função em termos dos campos vetoriais $\left\{X_{j}\right\}_{j=1}^{q}$, a saber,

$$
\left|f(x)-f_{B}\right| \leq C \int_{\tau B}|X f(y)| \frac{\rho(x, y)}{|B(x, \rho(x, y))|} d y, \quad x \in B,
$$

onde $C$ e $\tau$ são constantes positivas.

O primeiro passo para provar a fórmula de representação é apresentar uma estimativa pontual para o campo vetorial levantado $\left\{\widetilde{X_{j}}\right\}$, onde $\left\{\widetilde{X_{j}}\right\}$ é obtido da seguinte maneira: primeiro adicionamos novas variáveis, $\left(t_{1}, \ldots, t_{d}\right)=t \in \mathbb{R}^{d}$, a 
$\left(x_{1}, \ldots, x_{N}\right)=x \in \mathbb{R}^{N}$, e então obtemos um novo campo de vetores $\left\{\widetilde{X_{j}}\right\}$ em $\Omega \times \mathbb{R}^{d}$, o qual é dado por

$$
\left\langle\widetilde{X_{j}}, \nabla_{x, t}\right\rangle=\left\langle X_{j}, \nabla_{x}\right\rangle+\sum_{l=1}^{d} a_{j l}(x, t) \frac{\partial}{\partial t_{l}}, \quad j=1, \ldots, m,
$$

de tal forma que os novos campos de vetores $\left\{\widetilde{X_{\alpha}}\right\}_{|\alpha| \leq M}$ de comprimento no máximo $M$ gerem o espaço tangente $\mathbb{R}^{N+d}$ em cada ponto $(x, t) \in \Omega \times \mathbb{R}^{d}$.

A coleção $\left\{\widetilde{X_{j}}\right\}_{j=1}^{m}$ é definida como um levantamento ou campo vetorial livre. Se cada $m_{i}$ denota o número de comutadores de comprimento $i$ que são linearmente independentes (o comprimento de cada $\widetilde{X_{j}}$ é 1 ), então o número

$$
Q=\sum_{i=1}^{M} i m_{i}
$$

é chamado a dimensão homogênea do $\mathbb{R}^{N}$ com relação a $\left\{X_{j}\right\}$. No que segue, denotaremos $\widetilde{\Omega}=\Omega \times U_{0}$, onde $U_{0}$ é a bola unitária centrada na origem em $\mathbb{R}^{d}$, e $\widetilde{\rho}$ denotará a métrica sobre $\widetilde{\Omega} \times \widetilde{\Omega}$ associada aos campos de vetores $\left\{\widetilde{X}_{j}\right\}_{j=1}^{m}$. A bola métrica correspondente será denotada por $\widetilde{B}=\widetilde{B}(\xi, r)$, com $\xi \in \widetilde{\Omega}$.

Também necessitaremos das propriedades a seguir.

Dado um compacto $K \subset \Omega$ e $r_{0}>0$, temos

$$
|\widetilde{B}(\xi, r)| \approx r^{Q}
$$

com constantes de equivalência independentes de $\xi \in K \times U_{0}$ e $0<r<r_{0}$, como pode ser visto em (2.2) de [FLW2], à página 586.

E se $x \in K$, com $0<r<r_{0}$, então

$$
\widetilde{\rho}((x, s),(y, t)) \geq \rho(x, y),
$$

e

$$
\int_{\mathbb{R}^{d}} \chi_{\widetilde{B}((x, 0), r)}(y, t) d t \leq C \frac{|\widetilde{B}((x, 0), r)|}{|B(x, r)|},
$$

como pode se observado nos Lemas 3.1 e 3.2 de [NSW], à página 139

E agora podemos enunciar a estimativa pontual mencionada anteriormente (para detalhes ver páginas 586-589 de [FLW2]). 
Lema 4.5. Sejam $M \subset \widetilde{K}$ um subconjunto compacto de $\widetilde{\Omega}$ e $\widetilde{B}=\widetilde{B}\left(\xi_{0}, r\right)$ com $\xi_{0} \in \widetilde{K}, 0<r<r_{0}$. Então existem constantes $C$ e $c_{\widetilde{B}}$ tais que

$$
\left|f(\xi)-c_{\widetilde{B}}\right| \leq C \int_{C \widetilde{B}} \frac{(|\widetilde{X} f|+|f|)(\eta)}{\widetilde{\rho}(\xi, \eta)^{Q-1}} d \eta, \quad \xi \in \widetilde{B}
$$

para qualquer função $f \in \operatorname{Lip}(\overline{C \widetilde{B}})$, onde $C$ é independente de $f$ e $\widetilde{B}$, onde $|\widetilde{X} f|^{2}=$ $\sum_{j}\left\langle\widetilde{X_{j}}, \nabla f\right\rangle^{2}$

O próximo lema nos dá uma estimativa para o campo vetorial original.

Lema 4.6. Seja $K \subset \Omega$ um subconjunto compacto e $B=B\left(x_{0}, r\right)$ uma $\rho$-bola com $x_{0} \in K$ e $0<r$. Então

$$
\left|f(x)-f_{B}\right| \leq C \int_{C B}(|X f|+|f|)(y) \frac{\rho(x, y)}{|B(x, \rho(x, y))|} d y, \quad x \in B
$$

para toda $f \in \operatorname{Lip}(\overline{C B})$, onde $C$ independe de $f$ e de $B$, e $f_{B}=\frac{1}{|B|} \int_{B} f(y) d y$.

Prova: Primeiro mostraremos que a conclusão do lema também é válida para alguma constante $c_{B}$ no lugar de $f_{B}$. Para isso, utilizaremos o Lema 4.5. Sejam $B=B\left(x_{0}, r\right)$ e $\widetilde{B}=\widetilde{B}\left(\xi_{0}, r\right)$, onde $\xi_{0}=\left(x_{0}, 0\right)$. Note que $\widetilde{B} \subset B \times \mathbb{R}^{d}$, por (4.3). Estendendo $f$ ao fecho de $C \widetilde{B}$ fazendo $f$ constante em $t$, isto é, se $\xi=(x, t)$, então $f(\xi)=f(x)$, como $\tilde{X} f(\eta)=X f(y)$ se $\eta=(y, t)$, então pelo Lema 4.5 , temos

$$
\begin{aligned}
\left|f(x)-c_{\widetilde{B}}\right| & \leq C \int_{C \widetilde{B}} \frac{(|X f|+|f|)(y)}{\widetilde{\rho}((x, 0),(y, t))^{Q-1}} d y d t \quad x \in B \\
& =C \int_{\mathbb{R}^{N}}(|X f|+|f|)(y)\left\{\int_{\mathbb{R}^{d}} \chi_{C \widetilde{B}}(y, t) \frac{d t}{\widetilde{\rho}((x, 0),(y, t))^{Q-1}}\right\} d y \\
& \leq C \int_{C B}(|X f|+|f|)(y)\left\{\int_{\mathbb{R}^{d}} \frac{d t}{\widetilde{\rho}((x, 0),(y, t))^{Q-1}}\right\} d y,
\end{aligned}
$$

pois $C \widetilde{B} \subset C B \times \mathbb{R}^{d}$. Agora fixe $y$ e considere $\rho=\rho(x, y)$. Como $\widetilde{\rho}((x, 0),(y, t)) \geq$ $\rho(x, y)$ por $(4.3)$, temos as seguintes estimativas para a integral interna na expressão 
acima:

$$
\begin{aligned}
\int_{\mathbb{R}^{d}} \frac{d t}{\tilde{\rho}((x, 0),(y, t))^{Q-1}} & \leq \sum_{j=0}^{\infty} \frac{1}{\left(2^{j} \rho\right)^{Q-1}} \int_{2^{j} \rho \leq \widetilde{\rho}((x, 0),(y, t)) \leq 2^{j+1} \rho} d t \\
& \leq \sum_{j=0}^{\infty} \frac{1}{\left(2^{j} \rho\right)^{Q-1}} \int_{\mathbb{R}^{d}} \chi_{\widetilde{B}\left((x, 0), 2^{j+1} \rho\right)}(y, t) d t \\
& \leq C \sum_{j=0}^{\infty} \frac{1}{\left(2^{j} \rho\right)^{Q-1}} \frac{\left(2^{j+1} \rho\right)^{Q}}{\left|B\left(x, 2^{j+1} \rho\right)\right|}, \quad \text { por }(4.4) \text { e }(4.2) \\
& \leq C \sum_{j=0}^{\infty} \frac{2^{j} \rho}{\left|B\left(x, 2^{j+1} \rho\right)\right|} \\
& =C \sum_{j=0}^{\infty} \frac{2^{j} \rho}{|B(x, \rho)|} \frac{|B(x, \rho)|}{\left|B\left(x, 2^{j+1} \rho\right)\right|} \\
& \leq C \sum_{j=0}^{\infty} \frac{2^{j} \rho}{|B(x, \rho)|}\left(\frac{\rho}{2^{j+1} \rho}\right)^{N} \quad \text { por }(1.21) \operatorname{com} \alpha=N \\
& =C \sum_{j=0}^{\infty} 2^{j(1-N)} \frac{\rho}{|B(x, \rho)|},
\end{aligned}
$$

ou seja,

$$
\int_{\mathbb{R}^{d}} \frac{d t}{\widetilde{\rho}((x, 0),(y, t))^{Q-1}} \leq C \frac{\rho}{|B(x, \rho)|}
$$

pois $N>1$. Portanto, para $c_{B}=c_{\widetilde{B}}$, temos

$$
\left|f(x)-c_{B}\right| \leq C \int_{C B}(|X f|+|f|)(y) \frac{\rho(x, y)}{|B(x, \rho(x, y))|} d y, x \in B .
$$

E isso nos permite provar que $c_{B}$ pode ser trocado por $f_{B}=\frac{1}{|B|} \int_{B} f(z) d z$. De fato,

$$
\begin{aligned}
\left|f_{B}-c_{B}\right| & \leq \frac{1}{|B|} \int_{B}\left|f(z)-c_{B}\right| d z \\
& \leq \frac{1}{|B|} \int_{B}\left\{C \int_{c B}(|X f|+|f|)(y) \frac{\rho(x, y)}{|B(z, \rho(z, y))|} d z\right\} d y \text { por (4.7) } \\
& \leq C \int_{C B}(|X f|+|f|)(y)\left\{\frac{1}{|B|} \int_{B} \frac{\rho(z, y)}{|B(y, \rho(z, y))|} d z\right\} d y
\end{aligned}
$$

pois $\mid B(y, \rho(z, y) \mid$ e $\mid B(z, \rho(z, y) \mid$ são equivalentes, já que a medida de Lebesgue é dobrante. Como

$$
\left|f(x)-f_{B}\right| \leq\left|f(x)-c_{B}\right|+\left|f_{B}-c_{B}\right|
$$

é suficiente mostrar a seguinte relação:

$$
\frac{1}{|B|} \int_{B} \frac{\rho(z, y)}{|B(y, \rho(z, y))|} d z \leq C \frac{\rho(x, y)}{|B(y, \rho(x, y))|}, \quad x, y \in B .
$$


Para provar (4.8), fixe $y \in B$. Como $\rho(z, y) \leq C r(B)$ para $z \in B$, a expressão do lado esquerdo de (4.8) é no máximo

$$
\begin{aligned}
& \frac{1}{|B|} \sum_{j=0}^{\infty}\left(\int_{C 2^{-j} r(B) \leq \rho(z, y) \leq C 2^{1-j} r(B)} d z\right) \frac{C 2^{1-j} r(B)}{\left|B\left(y, C 2^{-j} r(B)\right)\right|} \\
& \leq \frac{1}{|B|} \sum_{j=0}^{\infty} \frac{\left|B\left(y, C 2^{1-j} r(B)\right)\right|}{\left|B\left(y, C 2^{-j} r(B)\right)\right|} C 2^{-j} r(B) \\
&=C \frac{r(B)}{|B|}, \text { por dobramento. }
\end{aligned}
$$

Portanto, se $x, y \in C B$, então $\rho(x, y) \leq C r(B)$, e por (1.21), com $\alpha=N$, podemos escrever

$$
\begin{aligned}
\frac{|B(x, \rho(x, y))|}{\rho(x, y)} & \leq C\left(\frac{\rho(x, y)}{r(B)}\right)^{N-1} \frac{|B(x, r(B))|}{r(B)} \\
& \leq C \frac{|B(x, r(B))|}{r(B)} \text { pois } N>1 \\
& \leq C \frac{|B|}{r(B)} \text { por dobramento. }
\end{aligned}
$$

Assim,

$$
\frac{1}{|B|} \int_{B} \frac{\rho(z, y)}{|B(y, \rho(z, y))|} \leq C \frac{r(B)}{|B|} \leq C \frac{\rho(x, y)}{|B(x, \rho(x, y))|}
$$

e portanto,

$$
\begin{aligned}
\left|f(x)-c_{B}\right| & \leq C \int_{C B}(|X f|+|f|)(y)\left\{\frac{1}{|B|} \int_{B} \frac{\rho(z, y)}{|B(y, \rho(z, y))|} d z\right\} d y \\
& \leq C \int_{C B}(|X f|+|f|)(y) \frac{\rho(x, y)}{|B(x, \rho(x, y))|} d y
\end{aligned}
$$

e o lema está provado.

Agora estamos preparados para provar a estimativa (4.1).

Proposição 4.10. Sejam $K \subset \Omega$ um subconjunto compacto e $B=B\left(x_{0}, r\right)$, com $x_{0} \in K$ e $0<r$. Existem constantes positivas $C$, c e $r_{0}$ dependendo somente de $K$, $\Omega$ e $\left\{X_{j}\right\}$ tais que se $k \in \mathbb{Z}$, e $S_{k}$ e $S_{k}^{*}$ são definidos por

$$
S_{k}=\left\{x \in B: 2^{k}<\left|f(x)-f_{B}\right| \leq 2^{k+1}\right\}
$$

e

$$
S_{k}^{*}=\left\{x \in c B: 2^{k}<\left|f(x)-f_{B}\right| \leq 2^{k+1}\right\},
$$


então

$$
\left|f(x)-f_{B}\right| \leq C \int_{\Omega}|X f| \chi_{S_{k-1}^{*}}(x) \frac{\rho(x, y)}{|B(x, \rho(x, y))|} d y+C \frac{r}{|B|} \int_{B}|X f| d y
$$

para todo $x \in S_{k}$ e toda $\left.f \in \operatorname{Lip} \overline{(C B}\right)$ e $0<r<r_{0}$. Além disso,

$$
\left|f(x)-f_{B}\right| \leq C \int_{c B}|X f| \frac{\rho(x, y)}{|B(x, \rho(x, y))|} d y, \quad x \in B .
$$

Prova: Para cada $x \in \Omega$, defina

$$
f_{k}(x)= \begin{cases}2^{k-1}, & \text { se }\left|f(x)-f_{B}\right| \leq 2^{k-1}, \\ \left|f(x)-f_{B}\right|, & \text { se } 2^{k-1}<\left|f(x)-f_{B}\right|<2^{k} \\ 2^{k}, & \text { se }\left|f(x)-f_{B}\right| \geq 2^{k} .\end{cases}
$$

Então $2^{k-1} \leq f_{k}(x) \leq 2^{k-1}+\left|f(x)-f_{B}\right|$. Assim, se $x \in S_{k}$,

$$
\begin{aligned}
2^{k}=f_{k}(x) & \leq\left|f_{k}(x)-\left(f_{k}\right)_{B}\right|+\left(f_{k}\right)_{B} \\
& \leq C \int_{c B}\left(\left|X f_{k}\right|+\left|f_{k}\right|\right)(y) \frac{\rho(x, y)}{|B(x, \rho(x, y))|} d y+2^{k-1} \\
& +\frac{1}{|B|} \int_{B}\left|f-f_{B}\right| d z \text { pelo Lema 4.6 } \\
& \leq C \int_{S_{k-1}^{*}}|X f(y)| \frac{\rho(x, y)}{|B(x, \rho(x, y))|} d y+\int_{c B}\left|f_{k}(y)\right| \frac{\rho(x, y)}{|B(x, \rho(x, y))|} d y \\
& +2^{k-1}+\frac{1}{|B|} \int_{B}\left|f-f_{B}\right| d z,
\end{aligned}
$$

pois $\left|X f_{k}\right| \chi_{c B} \leq|X f| \chi_{S_{k-1}^{*}}$. Como $2^{k-1} \leq f_{k} \leq 2^{k}$, obtemos

$$
\int_{c B} f_{k}(y) \frac{\rho(x, y)}{|B(x, \rho(x, y))|} d y \leq 2^{k} \int_{c B} \frac{\rho(x, y)}{|B(x, \rho(x, y))|} \leq C r 2^{k},
$$

onde $r=r(B)$, conforme (4.8).

Agora, aplicando a desigualdade de Poincaré para medida de Lebesgue e $p=$ $q=1,(\operatorname{veja}[\mathrm{J}])$, obtemos

$$
\frac{1}{|B|} \int_{B}\left|f(z)-f_{B}\right| d z \leq C \frac{r}{|B|} \int_{B}|X f(y)| d y .
$$

E combinando estas estimativas, para cada $x \in S_{k}$, temos que

$$
2^{k} \leq C \int_{S_{k-1}^{*}}|X f| \frac{\rho(x, y)}{|B(x, \rho(x, y))|} d y+C r 2^{k}+2^{k-1}+C \frac{r}{|B|} \int_{B}|X f| d y
$$


Como $C r 2^{k}<\frac{1}{3} 2^{k}$ quando $r$ é pequeno, e independente de $k$, obtemos por subtração que

$$
2^{k} \leq C \int_{S_{k-1}^{*}}|X f| \frac{\rho(x, y)}{|B(x, \rho(x, y))|} d y+C \frac{r}{|B|} \int_{B}|X f| d y,
$$

para $x \in S_{k}$ e $r$ pequenc.

Visto que $\left|f(x)-f_{B}\right| \leq 2^{k+1}$ para $x \in S_{k}$, obtemos (4.11).

E (4.12) segue de (4.11) e do fato de que

$$
\int_{S_{k-1}^{*}}|X f| \frac{\rho(x, y)}{|B(x, \rho(x, y))|} d y \leq C \int_{c B}|X f| \frac{\rho(x, y)}{|B(x, \rho(x, y))|} d y,
$$

já que $S_{k-1}^{*} \subset c B$, e por (4.9),

$$
\begin{aligned}
\frac{r}{|B|} \int_{B}|X f| d y & \leq C \int_{B}|X f(y)| \frac{\rho(x, y)}{|B(x, \rho(x, y))|} d y, \quad x \in B \\
& \leq C \int_{C B}|X f(y)| \frac{\rho(x, y)}{|B(x, \rho(x, y))|} d y, \quad x \in B
\end{aligned}
$$

Portanto,

$$
\left|f(x)-f_{B}\right| \leq C \int_{c B}|X f| \frac{\rho(x, y)}{|B(x, \rho(x, y))|} d y,
$$

para todo $x \in B$, e isso termina a demonstração.

Note que (3.18) também é válida, pois

$$
C \frac{\phi\left(B_{0}\right)}{\phi(B)}=C \frac{r\left(B_{0}\right)}{\left|B_{0}\right|} \frac{|B|}{r(B)} \geq \frac{|B|}{\left|B_{0}\right|}
$$

para toda bola $B$ tal que $\tau B \subset B_{0}$. Assim, (3.19) está satisfeita com a seguinte forma:

$$
\frac{1}{\left|B_{0}\right|} \int_{B_{0}}\left|f(x)-f_{B_{0}}\right| d x \leq C r\left(B_{0}\right)\left(\frac{1}{\left|B_{0}\right|} \int_{B_{0}}|X f(x)| d x\right) .
$$

\subsection{Equivalência da desigualdade de Poincaré e existência de fórmulas de representação para campos vetoriais de Grushin}

Sejam $X_{1}, \ldots, X_{N}$ campos vetoriais de Grushin, ou seja,

$$
X_{1}=\frac{\partial}{\partial x_{1}}, \ldots, X_{n}=\frac{\partial}{\partial x_{n}}, X_{n+1}=\lambda(x) \frac{\partial}{\partial y_{1}}, \ldots, X_{N}=\lambda(x) \frac{\partial}{\partial y_{m}},
$$


com $n, m \geq 1, n+m=N, \lambda \in C^{\infty}\left(\mathbb{R}^{n}\right)$. Consideremos $\rho$ a métrica associada a estes campos. Então $\left(\mathbb{R}^{N}, \rho, d x\right)$ é um espaço homogêneo.

Considerando $d \mu=\omega^{1-\frac{1}{N}} d x$, onde $\omega$ é um peso $A_{\infty}$-forte, pela desigualdade (2.30), temos

$$
\frac{r(B)}{\mu(B)}=\frac{r(B)}{\int_{B} \omega^{1-\frac{1}{N}}} \approx\left(\frac{1}{\int_{B} \omega(x) \lambda(x)^{\frac{m}{N-1}} d x}\right)^{1-\frac{1}{N}}
$$

e se $\widetilde{B} \subset B$, então por (ii) do Lema 2.10, temos que $\omega(x) \lambda(x)^{\frac{m}{N-1}}$ satisfaz a propriedade dobrante reversa, e portanto, por (i) do mesmo lema, existe $\delta>0$ tal que

$$
\int_{B} \omega(x) \lambda^{\frac{m}{N-1}}(x) d x \geq C\left(\frac{r(B)}{r(\widetilde{B})}\right)^{\delta} \int_{\widetilde{B}} \omega(x) \lambda(x)^{\frac{m}{N-1}} d x .
$$

Agora, combinando (4.14) com a equivalência (4.13) para $B$ e $\widetilde{B}$, obtemos (3.7) com $\varepsilon=\delta \frac{N-1}{N}$. De fato, por (4.13), temos

$$
c_{1} \int_{B} \omega(x) \lambda(x)^{\frac{m}{N-1}} d x \geq\left(\frac{\mu(B)}{r(B)}\right)^{\frac{N}{N-1}} \geq c_{2} \int_{B} \omega(x) \lambda(x)^{\frac{m}{N-1}} d x
$$

e o mesmo resultado para $\widetilde{B}$. Como $\frac{\epsilon}{\delta}=\frac{N-1}{N}$, combinando estes resultados com (4.14), segue que

$$
\begin{aligned}
\left(\frac{\mu(B)}{r(B)}\right)^{\frac{\delta}{\varepsilon}} & \geq C \int_{B} \omega(x) \lambda(x)^{\frac{m}{N-1}} d x \\
& \geq C\left(\frac{r(B)}{r(\widetilde{B})}\right)^{\delta} \int_{\widetilde{B}} \omega(x) \lambda(x)^{\frac{m}{N-1}} d x \\
& \geq C\left(\frac{r(B)}{r(\widetilde{B})}\right)^{\delta}\left(\frac{\mu(\widetilde{B})}{r(\widetilde{B})}\right)^{\frac{\delta}{\varepsilon}} .
\end{aligned}
$$

Elevando ambos os membros desta desigualdade a potência $\frac{\varepsilon}{\delta}$, obtemos

$$
\frac{\mu(B)}{r(B)} \geq C\left(\frac{r(B)}{r(\widetilde{B})}\right)^{\varepsilon}\left(\frac{\mu(\widetilde{B})}{r(\widetilde{B})}\right)
$$

ou seja,

$$
\mu(B) \geq C\left(\frac{r(B)}{r(\widetilde{B})}\right)^{\varepsilon+1} \mu(\widetilde{B})
$$

para toda bola $\widetilde{B} \subset B$, e portanto (3.7) está verificada. 
Se assumirmos (3.6) para $X=\nabla_{\lambda}(\operatorname{ver}[\mathrm{FGW}])$ para alguma medida dobrante $\nu$ e alguma bola $B_{0}$, ou seja, se assumirmos que

$$
\frac{1}{\nu(B)} \int_{B}\left|f-f_{B_{0}, \nu}\right| d \nu \leq C r(B) \frac{1}{\mu(B)} \int_{B}\left|\nabla_{\lambda} f\right| d \mu,
$$

para alguma bola $B_{0}$, então, pelo Teorema 3.5 podemos escrever

$$
\begin{aligned}
\left|f(x)-f_{B_{0}, \nu}\right| & \leq C \int_{\tau B}\left|\nabla_{\lambda} f(y)\right| \frac{\rho(x, y)}{\mu(B(x, \rho(x, y)))} d \mu \\
& =C \int_{\tau B}\left|\nabla_{\lambda} f(y)\right| \frac{\rho(x, y)}{\int_{B(x, \rho(x, y))} \omega^{1-\frac{1}{N}} d z} \omega^{1-\frac{1}{N}} d y
\end{aligned}
$$

$\nu$-quase sempre em $B_{0}, \operatorname{com} \tau>1$. Agora usando novamente (4.13), obtemos

$$
\left|f(x)-f_{B_{0}, \nu}\right| \leq C \int_{\tau B_{0}}\left|\nabla_{\lambda} f(y)\right| \frac{1}{\int_{B(x, \rho(x, y))} \omega \lambda^{\frac{m}{N-1}} d z} \omega^{1-\frac{1}{N}} d y,
$$

$\nu$-quase sempre em $B_{0}$.

E pelo Teorema 3.5, temos uma fórmula de representação neste caso, ou seja,

$$
\left|f(x)-f_{B_{0}, \nu}\right| \leq \int_{\tau k B_{0}}|X f(y)| \frac{\rho(x, y)}{\mu(B(x, \rho(x, y))) \mid} .
$$

Reciprocamente, mostraremos que pelo Teorema 3.14, a fórmula de representação acima implica a desigualdade de Poincaré (3.6) $\operatorname{com} d \mu=\omega^{1-\frac{1}{N}} d x$ e $d \nu=\omega^{1-\frac{1}{N}} d x$ ou $d \nu=\omega \lambda^{\frac{m}{N-1}} d x$. De fato, primeiro consideremos o caso em que

$$
d \mu=\omega^{1-\frac{1}{N}} d x, \quad \phi(B)=\frac{r(B)}{\mu(B)}, \quad \text { e } \quad d \sigma=\left|\nabla_{\lambda}\right| d \mu .
$$

Precisaremos da proposição a seguir, que é um resultado de [FGW], (ver página 574 para detalhes).

Proposição 4.15. Sejam $\omega$ um peso $A_{\infty}$-forte e $f$ uma função $C^{\infty}$. Então, dada uma bola $B$, existe uma constante $c_{B}=c(B, f)$ tal que para quase todo $x \in B$,

$$
\left|f(x)-c_{B}\right| \leq C \int_{\tau B}\left|\nabla_{\lambda} f(\xi)\right| \omega^{1-\frac{1}{N}}(\xi) K(x, \xi) d \xi
$$

onde $\tau>0$ e

$$
K(x, \xi)=\left(\int_{B(x, \rho(x, \xi))} \omega(y) \lambda(y)^{\frac{m}{N-1}} d y\right)^{\frac{1}{N}-1} .
$$

Então, por este resultado, temos que

$$
\begin{aligned}
\left|f(x)-c_{B}\right| & \leq C \int_{\tau B}\left|\nabla_{\lambda} f(\xi)\right| K(x, \xi) \omega^{1-\frac{1}{N}} d \xi \\
& =C \int_{\tau B}\left|\nabla_{\lambda} f(\xi)\right| K(x, \xi) d \mu(\xi) .
\end{aligned}
$$


Mas por (4.13) e a expressão de $K(x, \xi)$, podemos escrever

$$
\begin{aligned}
\left|f(x)-c_{B}\right| & \leq C \int_{\tau B}\left|\nabla_{\lambda} f(\xi)\right|\left(\int_{B(x, \rho(x, \xi))} \omega(y) \lambda(y)^{\frac{m}{N-1}} d y\right)^{\frac{1}{N}-1} d \mu \\
& =C \int_{\tau B}\left|\nabla_{\lambda} f(\xi)\right|\left(\frac{1}{\int_{B(x, \rho(x, \xi))} \omega(y) \lambda(y)^{\frac{m}{N-1}} d y}\right)^{1-\frac{1}{N}} d \mu \\
& \leq C \int_{\tau B}\left|\nabla_{\lambda} f(\xi)\right|\left(\frac{\rho(x, \xi)}{\int_{B(x, \rho(x, \xi))} \omega^{1-\frac{1}{N}} d y}\right) d \mu \quad \text { por }(4.13) \\
& =C \int_{\tau B}\left|\nabla_{\lambda} f(\xi)\right| \frac{\rho(x, \xi)}{\mu(B(x, \rho(x, \xi)))} d \mu \\
& =C \int_{\tau B} \phi(B(x, \rho(x, \xi))) d \sigma(y) .
\end{aligned}
$$

E portanto (3.15) está verificada.

Agora vamos mostrar que (3.16) e (3.18) são válidas para $d \nu=d \mu$ e $d \nu=$ $\omega \lambda^{\frac{m}{N-1}} d x$. Como $\mu$ é uma medida dobrante, temos

$$
\phi(B(x, \rho(x, y))) \lesssim \phi(B(y, \rho(x, y)))
$$

e portanto,

$$
\int_{B} \phi(B(x, \rho(x, y))) d \nu(x) \lesssim \int_{B} \phi(B(y, \rho(x, y))) d \nu(x)
$$

Se $y \in \tau B$, aumentando proporcionalmente o domínio de integração $B$, podemos assumir que $y$ é o centro de $B$. E a última integral é então no máximo

$$
\sum_{j=0}^{\infty} \int_{\left\{x: \rho(x, y) \approx 2^{-j} r(B)\right\}} \phi(B(y, \rho(x, y))) d \nu(x) \leq C \sum_{j=0}^{\infty} \phi\left(2^{-j} B\right) \nu\left(2^{-j} B\right) .
$$

Se $\nu=\mu$, esta soma é

$$
\begin{aligned}
C \sum_{j=0}^{\infty} \frac{2^{-j} r(B)}{\mu\left(2^{-j} B\right)} \mu\left(2^{-j} B\right) & =C \sum_{j=0}^{\infty} 2^{-j} r(B) \\
& =C r(B) \\
& =C\left(\frac{r(B)}{\mu(B)}\right) \mu(B) \\
& =C \phi(B) \nu(B),
\end{aligned}
$$

e portanto,

$$
\frac{1}{\nu(B)} \int_{B} \phi(B(x, \rho(x, y))) d \nu(x) \leq C \phi(B)
$$


ou seja, (3.16) está verificada com $d \nu=\omega^{1-\frac{1}{N}} d x$.

Se $d \nu=\omega \lambda^{\frac{m}{N-1}} d x$, então $\phi(B) \lesssim \nu(B)^{\frac{1}{N}-1}$, por (4.13), e assim,

$$
\begin{aligned}
\sum_{j=0}^{\infty} \phi\left(2^{-j} B\right) \nu\left(2^{-j} B\right) & \leq C \sum_{j=0}^{\infty} \nu\left(2^{-j} B\right)^{\frac{1}{N}-1} \nu\left(2^{-j} B\right) \\
& =C \sum_{j=0}^{\infty} \nu\left(2^{-j} B\right)^{\frac{1}{N}} \\
& \leq C \sum_{j=0}^{\infty}\left(2^{-j \varepsilon} \nu(B)\right)^{\frac{1}{N}}
\end{aligned}
$$

para algum $\varepsilon>0$ pela propriedade dobrante reversa de $\nu$. E portanto,

$$
\begin{aligned}
\int_{B} \phi(B(x, \rho(x, y))) d \nu(x) & \leq C \sum_{j=0}^{\infty}\left(\frac{1}{2^{\frac{\epsilon}{N}}}\right)^{j} \nu(B)^{\frac{1}{N}} \\
& =C \nu(B)^{\frac{1}{N}} \\
& =C \nu(B)^{\frac{1}{N}-1} \nu(B) \\
& \leq C \phi(B) \nu(B)
\end{aligned}
$$

E isso prova (3.16) também neste caso.

A estimativa (3.18) é óbvia se $d \nu=d \mu$.

Agora se $d \nu=\omega \lambda^{\frac{m}{N-1}} d x$, então por (4.13), (3.18) é equivalente a

$$
\frac{\nu(B)}{\nu\left(B_{0}\right)} \leq c\left(\frac{\nu(B)}{\nu\left(B_{0}\right)}\right)^{1-\frac{1}{N}}, \quad \text { para } B \subset B_{0} .
$$

Mas isso é imediato, pois $\nu(B) \leq \nu\left(B_{0}\right)$ se $B \subset B_{0}$.

Finalmente, como as bolas satisfazem a condição de Boman $\mathcal{B}(\tau, \varsigma)$, (ver comentários à página 592 de [FGW]), pelo Teorema 5.4 de [FGW], a fórmula de representação estudada naquele trabalho implica (3.6), para ambas as escolhas de $\nu$ com $d \mu=\omega^{1-\frac{1}{N}} d x$. 


\section{Referências Bibliográficas}

[FZ] Fernandes, J. C. D. and Zani, S. L., Desigualdades de Poincaré e Sobolev com pesos, IMPA, 1995.

[FGW] Frnachi, B., Gutierrez, C. E. and Wheeden, R. L., Weighted SobolevPoincaré inequalities for Grushin type operators, Comm. Partial Differential Equations 19 (1994), 523-604.

[FLW1] Franchi, B., Lu, G. and Wheeden, R. L., A relationship between Poincaré type inequalities and representation formulas in spaces of homogeneous type, Intenat. Math. Res. Notices 1 (1996), 1-14.

[FLW2] Franchi, B., Lu, G. and Wheeden, R. L., Representation formulas and weighted Poincaré inequalities for Hörmander vector fields, Ann. Inst. Fourier (Grenoble) 45 (1995), 577-604.

[H] Helgason, S., Differential geometry, Lie groups, and symmetric spaces, Academic Press, INC., 1978.

[J] Jerison, D., The Poincaré inequality for vector fields satisfying Hörmander's condition, Duke Math. J. 53 (1986), 503-523.

[L] Lu, G., Weighted Poicaré and Sobolev inequalities for vector fields satisfying Hörmander's conditions and applications, Revista Mat. Iberoamericana 8 (1992), 367-439.

[NSW] Nagel, A., Stein, E. M. and Wainger, S., Balls and metrics defined by vector fields I: Basic Properties, Acta Math. 155 (1985), 103-147.

[S] Stein, E. M., Harmonic Analysis: real-variable methods, orthogonality, and oscillatory integrals, Princeton University Press, 1993. 
[WZ] Wheeden, R.L. and Zygmund, A., Measure and Integral: An Introdution to Real Analysis, Marcel Dekker, Inc., New York, 1977. 


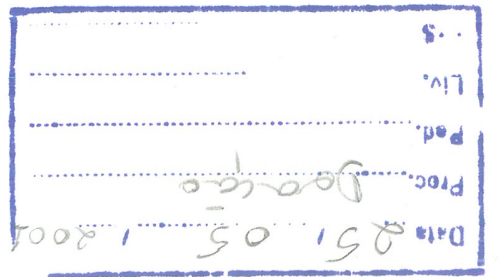

